\title{
Analysis of elastic symbols with the Cauchy integral and construction of asymptotic solutions
}

\author{
By Hideo SogA
}

(Received Sep. 5, 2016)

(Revised May 5, 2017)

\begin{abstract}
This paper deals with the elastic wave equation $\left(D_{t}^{2}-\right.$ $\left.L\left(x, D_{x^{\prime}}, D_{x_{n}}\right)\right) u\left(t, x^{\prime}, x_{n}\right)=0$ in the half-space $x_{n}>0$. In the constant coefficient case, it is known that the solution is represented by using the Cauchy integral $\int_{c} e^{i x_{n} \zeta}\left(I-L\left(\xi^{\prime}, \zeta\right)\right)^{-1} d \zeta$. In this paper this representation is extended to the variable coefficient case, and an asymptotic solution with the similar Cauchy integral is constructed. In this case, the terms $\partial_{x}^{\alpha} \int_{c} e^{i x_{n} \zeta}\left(I-L\left(x, \xi^{\prime}, \zeta\right)\right)^{-1} d \zeta$ appear in the inductive process. These do not become lower terms necessarily, and therefore the principal part of asymptotic solution is a little different from the form in the constant coefficient case.
\end{abstract}

\section{Introduction.}

In this paper we consider the elastic wave equation in the half-space $\mathbb{R}_{+}^{n}$ with the Dirichlet boundary condition:

$$
\begin{cases}\left(D_{t}^{2}-L\left(x, D_{x}\right)\right) u(t, x)=0 & \text { in } \mathbb{R} \times \mathbb{R}_{+}^{n}, \\ \left.u\right|_{x_{n}=0}=f\left(t, x^{\prime}\right) & \text { on } \mathbb{R} \times \mathbb{R}^{n-1},\end{cases}
$$

where $L\left(x, D_{x}\right)\left(x=\left(x^{\prime}, x_{n}\right)\right)$ is of the form

$$
L\left(x, D_{x}\right)=\sum_{j, l=1}^{n} a_{j l}(x)\left(-i \partial_{x_{j}}\right)\left(-i \partial_{x_{l}}\right)+\sum_{j=1}^{n} b_{j}(x)\left(-i \partial_{x_{j}}\right)+b_{0}(x) .
$$

The coefficients are $(n \times n)$-matrices of real-valued bounded $C^{\infty}$ functions with bounded derivatives (i.e. $\in \mathcal{B}^{\infty}\left(\overline{\mathbb{R}_{+}^{n}}\right)$ ). We assume that the principal part $L_{0}(x, \xi)$ of the symbol $L(x, \xi)$ satisfies the following conditions.

(A.1) $a_{j l}(x)={ }^{t} a_{l j}(x), \quad j, l=1,2, \ldots, n$.

(A.2) $L_{0}(x, \xi)$ is positive definite for any $(x, \xi) \in \overline{\mathbb{R}_{+}^{n}} \times \mathbb{R}^{n} \quad(\xi \neq 0)$.

(A.3) The multiplicity of every eigenvalue $\lambda_{j}(x, \xi)$ of $L_{0}(x, \xi)$ is constant.

$L_{0}(x, \xi)$ is a real symmetric matrix. The eigenvalues $\left\{\lambda_{j}(x, \xi)\right\}_{j=1, \ldots, d}$ are all positive and homogeneous of order 2 in $\xi$. Let $\lambda_{1}<\cdots<\lambda_{d}$. Fix $x \in \overline{\mathbb{R}}_{+}^{n}$ and $\eta^{\prime} \in \mathbb{R}^{n-1}$, and consider the equation (in $z$ )

2010 Mathematics Subject Classification. Primary 74B05; Secondary 35C20, 74J05, 35L05, 35L51.

Key Words and Phrases. elastic equations, wave equations, asymptotic solutions, singularities. 


$$
\operatorname{det}\left(I-L_{0}\left(x, \eta^{\prime}, z\right)\right)=0 .
$$

Then the real number $\tilde{z}$ is the root of this equation if and only if $\tilde{z} \operatorname{satisfies~} 1-\lambda_{j}\left(x, \eta^{\prime}, \tilde{z}\right)=$ 0 for some $j$.

We say that $\left(\tilde{x}^{\prime}, \tilde{\eta}^{\prime}\right)$ is non-glancing if $\partial_{\xi_{n}} \lambda_{j}\left(\tilde{x}^{\prime}, 0, \tilde{\eta}^{\prime}, \tilde{z}\right) \neq 0$ for any real $\tilde{z}$ with $\lambda_{j}\left(\tilde{x}^{\prime}, 0, \tilde{\eta}^{\prime}, \tilde{z}\right)=1$ for all $j(=1, \ldots, d)$. Let us say that the boundary value $f$ in $(1.1)$ is non-glancing if its wave front set $(\mathrm{WF}[f])$ is included in a conic neighborhood consisting of non-glancing points (for the definition of WF $[f]$, e.g., see Section 3 in Chapter 10 of Kumano-go $[\mathbf{3}]$ ).

Let $\left(\tilde{x}^{\prime}, \tilde{\eta}^{\prime}\right)$ be non-glancing. Then the number of the real roots $\tilde{z}$ of (1.2) with $\partial_{\xi_{n}} \lambda_{j}\left(x^{\prime}, 0, \eta^{\prime}, \tilde{z}\right)>0$ and the one of the roots with $\partial_{\xi_{n}} \lambda_{j}\left(x^{\prime}, 0, \eta^{\prime}, \tilde{z}\right)<0$ are same in a neighborhood $U^{\prime}$ of $\left(\tilde{x}^{\prime}, \tilde{\eta}^{\prime}\right)$. We assume that for any non-glancing point $\left(\tilde{x}^{\prime}, \tilde{\eta}^{\prime}\right)$

(A.4) there exists only one or no real point $\tilde{z}$ satisfying $1-\lambda_{j}\left(x^{\prime}, 0, \eta^{\prime}, \tilde{z}\right)=0$ with $\partial_{\xi_{n}} \lambda_{j}\left(x^{\prime}, 0, \eta^{\prime}, \tilde{z}\right)<0$ (when $\tilde{z}$ is real) for every $j$.

The multiplicity of the root $\tilde{z}$ coincides with the one of $\lambda_{j}\left(x, \eta^{\prime}, \tilde{z}\right)$. Let us note that the assumption (A.4) is satisfied if the slowness surface $\left\{\xi \mid \lambda_{j}(x, \xi)=1\right\}$ is strictly convex.

Under the assumptions (A.1)-(A.4) we classify the (distinct) $\operatorname{roots}\left\{z_{ \pm}^{j}\right\}_{j=1, \ldots, d}$ of (1.2) in the following way for $\left(x^{\prime}, \eta^{\prime}\right) \in U^{\prime}$ and $x_{n} \in J(J$ is a small interval $[0, r])$ :

$z_{+}^{j}\left(\operatorname{resp} . z_{-}^{j}\right)(j=1, \ldots, k)$ are real roots satisfying $1-\lambda_{j}\left(x, \eta^{\prime}, z_{ \pm}^{j}\right)=0$ and $\partial_{\xi_{n}} \lambda_{j}\left(x, \eta^{\prime}, z_{+}^{j}\right)<0$ (resp. $\left.>0\right)$;

$z_{ \pm}^{j}(j=k+1, \ldots, d)$ are non-real roots satisfying $z_{+}^{j}=\overline{z_{-}^{j}}$ and $\operatorname{Im} z_{+}^{j}>0$.

We assume that there exist at least two real roots (i.e., all the roots are not non-real). For the real roots $z_{ \pm}^{j}$ their multiplicities (mult $\left[z_{ \pm}^{j}\right]$ ) are equal to $\operatorname{dim} \operatorname{Ker}\left[I-L_{0}\left(x, \eta^{\prime}, z_{ \pm}^{j}\right)\right]$, and for the non-real roots $z_{ \pm}^{j}\left(x, \eta^{\prime}\right)$ it holds generally only that $\operatorname{dim} \operatorname{Ker}\left[I-L_{0}\left(x, \eta^{\prime}, z_{ \pm}^{j}\right)\right] \leq$ mult $\left[z_{ \pm}^{j}\right]$ (cf. Section 2 of Soga [6]). We assume that for $\left(x, \eta^{\prime}\right) \in U\left(=U^{\prime} \times J\right)$ every non-real root $z_{ \pm}^{j}\left(x, \eta^{\prime}\right)$ can be extended $C^{\infty}$-smoothly for the complex variable $\eta^{\prime}$ near the real values, and that for $j=k+1, \ldots, d$

(A.5) the multiplicity of $z_{ \pm}^{j}\left(x, \eta^{\prime}\right)$ is constant and is equal to $\operatorname{dim} \operatorname{Ker}\left[I-L_{0}\left(x, \eta^{\prime}, z_{ \pm}^{j}\right)\right]$.

In this paper we construct outgoing asymptotic solutions of (1.1) for non-glancing boundary values $f$. 'Outgoing' means that $\operatorname{sing} \operatorname{supp}[u] \subset\left\{t \geq t_{0}\right\}$ holds if $\operatorname{sing} \operatorname{supp}[f] \subset$ $\left\{t \geq t_{0}\right\}$. When the coefficients of $L_{0}$ are constant and $L=L_{0}$, we have some result due to Kawashita, Soga and Ralston [2]. In this case we expect that the function

$$
\iint e^{i \sigma t} e^{i x^{\prime} \xi^{\prime}}\left[\int_{c} e^{i x_{n} \zeta}\left(\sigma^{2} I-L_{0}\left(\xi^{\prime}, \zeta\right)\right)^{-1} \overline{d \zeta}\right] h\left(\sigma, \xi^{\prime}\right) \hat{f}\left(\sigma, \xi^{\prime}\right) \bar{d} \sigma \bar{d} \xi^{\prime}
$$

becomes an outgoing solution of (1.1), where $\hat{f}$ is the Fourier transform of $f$ in $\left(t, x^{\prime}\right)$, $\bar{d} \zeta=(2 \pi i)^{-1} d \zeta, \bar{d} \sigma=(2 \pi)^{-1} d \sigma$ and $\bar{d} \xi^{\prime}=(2 \pi)^{1-n} d \xi^{\prime}$. They in [2] show under some additional assumptions that this expectation is correct for some matrix $h$ and some path $c$. 
In the present paper we extend the result in $[\mathbf{2}]$ to the case of the variable coefficients. Namely, we construct the asymptotic solution with the integral terms of the same type as (1.3). To do so, we derive various properties of the Cauchy integrals $\int_{c} e^{i x_{n} \zeta} g\left(\sigma^{2} I-\right.$ $\left.L_{0}\left(x, \xi^{\prime}, \zeta\right)\right)^{-1} h d \zeta$ in Section 2. Firstly we need to check whether the derivatives of those integrals are also of the same type since their derivatives appear in the process of the construction (cf. Corollary 2.3). Let us note that the integral $\int_{c} e^{i x_{n} \zeta}\left(\sigma^{2} I-L_{0}\left(\xi^{\prime}, \zeta\right)\right)^{-1} d \zeta$ is transformed to the form $\int_{c} e^{i \sigma x_{n} z}\left(I-L_{0}\left(x, \eta^{\prime}, z\right)\right)^{-1} d z$ by change of the variables: $\xi^{\prime} \mapsto \sigma \eta^{\prime}$ and $\zeta \mapsto \sigma z(\sigma>0)$. We define the integral operators $\mathrm{Op}\left[\int_{c} e^{i \sigma x_{n}(z+\psi)} \cdots \bar{d} z\right]$ by

$$
\begin{aligned}
\mathrm{Op} & {\left[\int_{c} e^{i \sigma x_{n}(z+\psi)} g\left(I-L_{0}(z)\right)^{-1} h d z\right] f } \\
= & \int_{0}^{+\infty} e^{i \sigma t} \int e^{i \sigma x^{\prime} \eta^{\prime}}\left[\int_{c} e^{i \sigma x_{n}\left(z+\psi\left(x, \eta^{\prime}\right)\right)} g\left(x, \eta^{\prime}, z\right)\left(I-L_{0}\left(x, \eta^{\prime}, z\right)\right)^{-1} h\left(x, \eta^{\prime}\right) \bar{d} z\right] \\
& \hat{f}\left(\sigma, \sigma \eta^{\prime}\right) \chi\left(x, \sigma, \eta^{\prime}\right) \bar{d} \eta^{\prime} \sigma^{n-1} d \sigma,
\end{aligned}
$$

where $\chi\left(x, \sigma, \eta^{\prime}\right)$ is a $C^{\infty}$ cutoff function equal to 1 on $\tilde{U} \times\{\sigma>1\}$ for a neighborhood $\tilde{U}$ $(\subset U)$ and with $\operatorname{supp}_{\left(x, \eta^{\prime}\right)}[\chi] \subset U$. We omit notations of the variables $x$ or $\eta^{\prime}$ (e.g., $\left.L_{0}(z)\right)$ if confusion does not occur. $\left(I-L_{0}(z)\right)^{-1}$ has (simple) poles only at $z=z_{ \pm}^{j}(j=1, \ldots, d)$ (cf. Lemma 2.1 in Section 2). We can assume that $\chi\left(x, \sigma, \eta^{\prime}\right)=0$ near $\sigma=0$ in (1.4).

We expect that we can make the asymptotic solutions so that the principal term is of the same form as in the constant coefficient case (i.e., $\mathrm{Op}\left[\int_{c} e^{i \sigma x_{n} z} g\left(I-L_{0}(z)\right)^{-1} h \bar{d} z\right] f$ for some $g$ and $\left.h \in \mathcal{B}^{\infty}\left(\overline{\mathbb{R}_{+}^{n}} \times \mathbb{R}^{n-1}\right)\right)$. However, this is difficult. The residue of the integral $\int_{c} e^{i \sigma x_{n} z} g\left(I-L_{0}(z)\right)^{-1} h \bar{d} z$ is sum of the forms $e^{i \sigma x_{n} z_{+}^{j}} g\left\{\operatorname{Res}_{z=z_{+}^{j}}\left(I-L_{0}(z)\right)^{-1}\right\} h$. This means that the phase functions $x_{n} z_{+}^{j}$ are of the special form (i.e., product of $x_{n}$ and $z_{+}^{j}$ ). On the other hand, Soga [6] constructed the similar solutions by different procedures. The phase functions in [6] have more general forms in the Taylor expansion for $x_{n}$. This is not consistent with the above mention. It is also another difficulty in the variable coefficient case that the order of the pole increases when the derivation $\partial_{x}$ is applied to $\left(I-L_{0}\left(x, \eta^{\prime}, z\right)\right)^{-1}$ (in detail, cf. Section 2).

Therefore, we construct asymptotic solutions with several kinds of the phase functions $\sigma x_{n}\left(z+\psi\left(x, \eta^{\prime}\right)\right)$ in the Cauchy integral $\int_{c} e^{i \sigma x_{n}(z+\psi)} g\left(I-L_{0}\left(x, \eta^{\prime} z\right)\right)^{-1} h \bar{d} z$. Furthermore, we need to provide the integral with more than one kind of $g$ for each phase function. The reason for this is explained later. In this way we can construct the outgoing asymptotic solutions as stated in Theorem 1 below.

Let $H^{l}\left(\mathbb{R} \times \mathbb{R}^{n-1}\right)\left(=H^{l}\right)$ be the Sobolev space on $\mathbb{R}_{t} \times \mathbb{R}_{x^{\prime}}^{n-1}$ of order $l$. For non-negative integers $m$ and $s \in \mathbb{R}$ let $C^{m}\left(J ; H^{s}\right)$ denote the set of all $H^{s}$-valued $C^{m}$ functions on an interval $J=[0, r]$ in $\mathbb{R}_{x_{n}}$. We set

$$
\mathcal{H}^{m, s}=\bigcup_{l=0}^{m} C^{l}\left(J ; H^{s-l}\right) .
$$

$\mathrm{Op}\left[\int_{c} e^{i \sigma x_{n}(z+\psi)} g(z)\left(I-L_{0}(z)\right)^{-1} h \sigma^{-m} \bar{d} z\right]$ is a bounded linear operator from $H^{s}(s=$ $0,1, \ldots)$ to $\mathcal{H}^{s, s+m}$ if $\operatorname{Im}(z+\psi)$ is greater than 0 at every pole of $g(z)\left(I-L_{0}(z)\right)^{-1}$. If we obtain the outgoing solution in an interval $0 \leq x_{n} \leq r$, we can know about the singular- 
ities on the whole interval $0 \leq x_{n}<+\infty$ in a restricted interval in $t$ (cf. Corollary 6.3). We say that the formal sum $\sum_{m=0}^{\infty} u_{m}(t, x)\left(u_{m} \in \mathcal{H}^{2, m+2}\right)$ is an asymptotic solution of (1.1) if $\left(D_{t}^{2}-L\right) \sum_{m=0}^{N} u_{m} \in \mathcal{H}^{0, N-1}$ and $\left.\sum_{m=0}^{N} u_{m}\right|_{x_{n}=0}-f \in H^{N+1}$ for any positive integer $N$.

THEOREM 1. Let $\left(\tilde{x}^{\prime}, \tilde{\eta}^{\prime}\right)$ be non-glancing, and let $U^{\prime}$ be a small neighborhood of $\left(\tilde{x}^{\prime}, \tilde{\eta}^{\prime}\right)$. We take a small closed path $c_{+}$surrounding only the roots $z_{+}^{j}(j=1, \ldots, d)$, and take an interval $J$ small enough. Then, there exist $C^{\infty}$ functions $\psi^{j l}\left(x, \eta^{\prime}\right)(j, l=$ $1, \ldots, d), g^{l}\left(x, \eta^{\prime}, z\right)$ (analytic in $\left.z\right), h_{m}^{j}\left(x, \eta^{\prime}\right)(m=0,1, \ldots)$ and a neighborhood $\tilde{U}^{\prime}$ $\left(\subset U^{\prime}\right)$ such that for any $f\left(t, x^{\prime}\right)$ with $\mathrm{WF}[f] \subset\left\{\left(t, x^{\prime}, \sigma, \xi^{\prime}\right) \mid\left(x^{\prime}, \xi^{\prime} / \sigma\right) \in \tilde{U}^{\prime}, \sigma>0\right\}$

$$
\sum_{j=1}^{d} \sum_{l=1}^{d} \mathrm{Op}\left[\int_{c_{+}} e^{i \sigma x_{n}\left(z+\psi^{j l}\left(x, \eta^{\prime}\right)\right)} g^{l}\left(x, \eta^{\prime}, z\right)\left(I-L_{0}\left(x, \eta^{\prime}, z\right)\right)^{-1} \sum_{m=0}^{\infty} h_{m}^{j}\left(x, \eta^{\prime}\right) \sigma^{-m} \overline{d z}\right] f
$$

is an outgoing asymptotic solution of (1.1) for $x_{n} \in J$. Furthermore, the principal part is reformed in the form

$$
\sum_{j=1}^{d} \mathrm{Op}\left[\int_{c_{+}} e^{i \sigma x_{n}\left(z+\varphi^{j}\left(x, \eta^{\prime}\right)\right)} \tilde{g}_{0}^{j}\left(x, \eta^{\prime}, z\right)\left(I-L_{0}\left(x, \eta^{\prime}, z\right)\right)^{-1} \tilde{h}_{0}^{j}\left(x, \eta^{\prime}\right) \bar{d} z\right] f,
$$

where $\tilde{g}_{0}^{j}(z)$ is analytic and equal to 0 at $z=z_{+}^{l}$ for every $l \neq j$. The above functions $\varphi^{j}\left(x, \eta^{\prime}\right)$ and $\psi^{j l}\left(x, \eta^{\prime}\right)$ satisfy

$$
\left.\varphi^{j}\right|_{x_{n}=0}=0, \quad \psi^{j l}=z_{+}^{j}+\varphi^{j}-z_{+}^{l} \quad(j, l=1, \ldots, d) .
$$

Introduce operators of the form $\mathrm{Op}_{-}\left[\int_{c_{-}} e^{i \sigma x_{n}(z+\psi)} g(z)\left(I-L_{0}(z)\right)^{-1} h \bar{d} z\right] f=$ $\int_{-\infty}^{0} e^{i \sigma t} \int e^{i \sigma x^{\prime} \eta^{\prime}}\left[\int_{c_{-}} e^{i \sigma x_{n}(z+\psi)} g(z)\left(I-L_{0}(z)\right)^{-1} h \bar{d} z\right] \hat{f}\left(\sigma, \sigma \eta^{\prime}\right) \chi \bar{d} \eta^{\prime}|\sigma|^{n-1} \bar{d} \sigma$ with a path $c_{-}$surrounding only $z_{+}^{j}(j=1, \ldots, k)$ and $z_{-}^{j}(j=k+1, \ldots, d)$. Then, substituting $\mathrm{Op}_{-}\left[\int_{c_{-}} \cdots \overline{d z}\right]$ for $\mathrm{Op}\left[\int_{c_{+}} \cdots \bar{d} z\right]$, we can obtain the same theorem for $f$ with $\mathrm{WF}[f] \subset\left\{\left(t, x^{\prime}, \sigma, \xi^{\prime}\right) \mid\left(x^{\prime}, \xi^{\prime} / \sigma\right) \in \tilde{U}^{\prime}, \sigma<0\right\}$ as Theorem 1. Therefore, for any $f$ with $\mathrm{WF}[f] \subset\left\{\left(t, x^{\prime}, \sigma, \xi^{\prime}\right) \mid\left(x^{\prime}, \xi^{\prime} / \sigma\right) \in \tilde{U}^{\prime}\right\}$ we can construct the outgoing asymptotic solution consisting of sum of the parts $\mathrm{Op}\left[\int_{c_{+}} \cdots \overline{d z}\right]$ and $\mathrm{Op}_{-}\left[\int_{c_{-}} \cdots \overline{d z}\right]$.

In Theorem 1 the term $\sum_{l=1}^{d} \mathrm{Op}\left[\int_{c_{+}} e^{i \sigma x_{n}\left(z+\psi^{j l}\right)} g^{l}(z)\left(I-L_{0}(z)\right)^{-1} \sum_{m=0}^{\infty} h_{m}^{j}\right.$ $\left.\sigma^{-m} \bar{d} z\right] f$ for $j=1, \ldots, k$ represents the $\left(\lambda_{j}\right.$-mode) body wave, and the one for $j=k+1, \ldots, d$ does the surface wave. The body wave and the surface wave are associated with the real roots $z_{+}^{j}(j=1, \ldots, k)$ and the non-real roots $z_{+}^{j}(j=k+1, \ldots, d)$ respectively. Those constructions are also a little different each other (cf. Section 5 and Section 4 respectively).

Theorem 1 means that the lower terms (i.e. with the index $m=1,2, \ldots$ ) for each $j$ are sum of more than one term with the index $l=1, \ldots, d$. This is natural in view of the result in Soga $[6] . \operatorname{Res}_{z=z_{+}^{j}}\left(I-L_{0}(z)\right)^{-1}$ projects $\mathbb{C}^{n}$ to the space $\operatorname{Ker}\left[I-L_{0}\left(z_{+}^{j}\right)\right]$. On the other hand the result in $[\mathbf{6}]$ shows that the lower terms are not necessarily restricted in such a particular subspace as $\operatorname{Ker}\left[I-L_{0}\left(z_{+}^{j}\right)\right]$. Therefore, we provide some more than one term for each $j$, and sum them up (in the index $l$ ). In this procedure we need to show 
that $g^{l}$ can be chosen such that $\sum_{l=1}^{d} \int_{c_{+}} g^{l}(z)\left(I-L_{0}(z)\right)^{-1} \bar{d} z=I$. This is guaranteed by the following theorem.

THEOREM 2. Let $\left(\tilde{x}^{\prime}, \tilde{\eta}^{\prime}\right)$ be non-glancing. Then there exist $(n \times n)$-matrices $M^{j}\left(x, \eta^{\prime}\right) \in \mathcal{B}^{\infty}\left(\overline{\mathbb{R}_{+}^{n}} \times \mathbb{R}^{n-1}\right)$ and a neighborhood $U$ of $\left(\tilde{x}^{\prime}, 0, \tilde{\eta}^{\prime}\right)$ such that for $\left(x, \eta^{\prime}\right)$ $\in U$

$$
\sum_{j=1}^{d} M^{j}\left(x, \eta^{\prime}\right)\left\{\operatorname{Res}_{z=z_{+}^{j}}\left(I-L_{0}\left(x, \eta^{\prime}, z\right)\right)^{-1}\right\}=I .
$$

Noting that $\operatorname{Res}_{z=z_{+}^{j}}\left(I-L_{0}(z)\right)^{-1} \mathbb{C}^{n}=\operatorname{Ker}\left[I-L_{0}\left(z_{+}^{j}\right)\right]$ (cf. Lemma 2.1 in Section 2), we can derive this theorem from

$$
\operatorname{Ker}\left[I-L_{0}\left(x, \eta^{\prime}, z_{+}^{1}\right)\right]+\cdots+\operatorname{Ker}\left[I-L_{0}\left(x, \eta^{\prime}, z_{+}^{d}\right)\right]=\mathbb{C}^{n} .
$$

The above equality is stated also in Soga [6]. The proof in [6], however, contains an incomplete part. In Section 2 we prove (1.5) by the method different from the one in [6]. Our proof is an improvement of the method for Theorem 2.1 in Kawashita, Soga and Ralston [2].

Taylor [7] and others construct asymptotic solutions for more general equations similar to ours. We note that in the general cases such conditions as (1.5) do not necessarily hold and that (1.5) is due to the elasticity (i.e., (A.1)-(A.5)). The equality (1.5) can be checked rather easily when the surface waves do not appear (i.e., all the roots $z_{+}^{j}$ are real), which was shown in Soga [5]. In this case he also constructed the asymptotic solution by the same idea as in Soga $[6]$.

Using the asymptotic solution in this paper, we can know how the singularities of the outgoing (genuine) solution propagate near the boundary as $t(>0)$ moves near 0 (cf. Theorem 6.2). Our solution is essentially equal to the one in the paper of Soga [6] if the residue is taken in the integral $\int_{c_{+}} e^{i \sigma x_{n}\left(z+\psi^{j l}\right)} g^{l}\left(I-L_{0}(z)\right)^{-1} h_{m}^{j} d z$. Kumano-go [3] examines singularities of the Fourier integral operators of the general type. Adding concrete calculation to the results in $[\mathbf{6}]$ and $[\mathbf{3}]$, we can obtain the same informations of the singularities that in Section 6 . However, we remark that our asymptotic solution gives an integral form common to all the modes (i.e., all the parts associated with each of the roots $z_{+}^{j}$ ) with accuracy equivalent to one in $[\mathbf{6}]$, etc.

Outline of the paper is as follows: In the next section (Section 2) we analyze the integral $\int_{c} e^{i \sigma x_{n}(z+\psi)} g\left(I-L_{0}(z)\right)^{-1} h \bar{d} z$ and explain the basic properties. They are used later for the proof of above Theorem 1, etc. Section 3 is devoted to basic examination for the construction of the asymptotic solutions. In Section 4 the construction is performed in the case of the non-real root $z_{+}^{j}$. This is similar essentially to the Poisson operator. In Section 5 we construct the asymptotic solutions in the case of the real root $z_{+}^{j}$, and prove Theorem 1. The case of the real root $z_{+}^{j}$ is related with the Fourier integral operator. In Section 6 we examine the singularities of the solutions, and confirm the outgoingness of the solutions.

ACKNOWLEDGEMENTs. The author would thank the referee for his useful indications, which have made the descriptions and considerations more appropriate. 


\section{Analysis of the symbols.}

In this section we explain properties of the Cauchy integrals $\int_{c_{+}} e^{i \sigma x_{n}(z+\psi)} g(I-$ $\left.L_{0}(z)\right)^{-1} h \bar{d} z$. It is seen from the examination in this section that the set consisting of sum of those integrals is closed for differentiation (cf. Corollary 2.3). Throughout this section let the assumptions (A.1)-(A.5) in Section 1 be satisfied for a non-glancing point $\left(\tilde{x}^{\prime}, \tilde{\eta}^{\prime}\right)$, and let $U^{\prime}$ be a small neighborhood of $\left(\tilde{x}^{\prime}, \tilde{\eta}^{\prime}\right)$ and $J$ be a small interval $[0, r]$ in $\mathbb{R}_{x_{n}}$.

We see that a real value $\tilde{z}$ becomes a root of the equation (1.2) (i.e., $\operatorname{det}[I-$ $\left.\left.L_{0}\left(x, \eta^{\prime}, z\right)\right]=0,\left(x, \eta^{\prime}\right) \in U=U^{\prime} \times J\right)$ if and only if $\tilde{z}$ satisfies $1-\lambda_{j}\left(x, \eta^{\prime}, \tilde{z}\right)=0$ for some $j$. Furthermore, this $j$ is only one; because $\operatorname{det}\left[I-L_{0}(z)\right]=\left(1-\lambda_{1}(z)\right)^{\operatorname{mult}\left[\lambda_{1}\right] \ldots(1-}$ $\left.\lambda_{d}(z)\right)^{\text {mult }\left[\lambda_{d}\right]}$ and $\lambda_{1}(z), \ldots, \lambda_{d}(z)$ are distinct each other. The number $d_{+}^{j}$ of the $\tilde{z}$ with $\partial_{\xi_{n}} \lambda_{j}(\tilde{z})<0$ is equal to the one $d_{-}^{j}$ with $\partial_{\xi_{n}} \lambda_{j}(\tilde{z})>0$.

Let us verify this fact: Such numbers $d_{ \pm}^{j}$ are finite. Let $z_{1}<\cdots<z_{m}$ be all the real values $z$ satisfying $1-\lambda_{j}(z)=0$ (where $j$ is fixed). Since $\lim _{z \rightarrow-\infty} \lambda_{j}(z)=\infty$, the inequality $\partial_{\xi_{n}} \lambda_{j}\left(z_{1}\right)<0$ must hold. Therefore, $\left(1-\lambda_{j}(z)\right)>0$ on $\left(z_{1}, z_{2}\right)$. Hence, we have $\partial_{\xi_{n}} \lambda_{j}\left(z_{2}\right)>0$ and $\left(1-\lambda_{j}(z)\right)<0$ on $\left(z_{2}, z_{3}\right)$. Repeating this process inductively, we see that $m$ is an even integer and that $\partial_{\xi_{n}} \lambda_{j}\left(z_{l}\right)<0$ for the odd $l$ and $\partial_{\xi_{n}} \lambda_{j}\left(z_{l}\right)>0$ for the even $l$. Therefore we have $d_{+}^{j}=d_{-}^{j}$. Hereafter we assume that $d_{+}^{j}=d_{-}^{j}=1$, as is stated in (A.4) in Section 1.

We state some properties concerning the behavior of $\left(I-L_{0}\left(x, \eta^{\prime}, z\right)\right)^{-1}$ :

LEMMA 2.1. $\left(I-L_{0}\left(x, \eta^{\prime}, z\right)\right)^{-1}$ is a (matrix-valued) meromorphic function in $z$ and may have poles only at $\left\{z_{ \pm}^{j}\right\}_{j=1, \ldots, d}$. The following (i)-(iii) hold for $\left(x, \eta^{\prime}\right) \in U$ $\left(=U^{\prime} \times J\right)$ at $z=z_{+}^{j}$, and also the same ones hold at $z_{-}^{j}$.

(i) $\left(I-L_{0}\left(x, \eta^{\prime}, z\right)\right)^{-1}$ has a simple pole at $z_{+}^{j}$; namely, it is expanded at $z=z_{+}^{j}\left(x, \eta^{\prime}\right)$ :

$$
\left(I-L_{0}\left(x, \eta^{\prime}, z\right)\right)^{-1}=\frac{1}{z-z_{+}^{j}} R_{0}^{j}\left(x, \eta^{\prime}\right)+R_{1}^{j}\left(x, \eta^{\prime}\right)+\left(z-z_{+}^{j}\right) R_{2}^{j}\left(x, \eta^{\prime}\right)+\cdots
$$

(ii) $\left\{\operatorname{Res}_{z=z_{+}^{j}}\left(I-L_{0}\left(x, \eta^{\prime}, z\right)\right)^{-1}\right\} \mathbb{C}^{n}\left(=R_{0}^{j} \mathbb{C}^{n}\right)=\operatorname{Ker}\left[I-L_{0}\left(x, \eta^{\prime}, z_{+}^{j}\right)\right]$.

(iii) Let $z_{+}^{j}$ be the real root and denote by $P^{j}\left(x, \eta^{\prime}\right)$ the orthogonal projection to the eigen-space $\left(=\operatorname{Ker}\left[I-L_{0}\left(x, \eta^{\prime}, z_{+}^{j}\right)\right]\right)$ of the eigen-value $\lambda_{j}\left(x, \eta^{\prime}, z_{+}^{j}\right)$. Then we have

$$
\operatorname{Res}_{z=z_{+}^{j}}\left(I-L_{0}\left(x, \eta^{\prime}, z\right)\right)^{-1}\left(=R_{0}^{j}\right)=\left(\partial_{\xi_{n}} \lambda_{j}\left(x, \eta^{\prime}, z_{+}^{j}\right)\right)^{-1} P^{j}\left(x, \eta^{\prime}\right) .
$$

Noting that $\left(I-L_{0}(z)\right)^{-1}=\left(\operatorname{det}\left[I-L_{0}(z)\right]\right)^{-1} \operatorname{cof}\left[I-L_{0}(z)\right](\operatorname{cof}[\cdot]$ denotes the cofactor), we see easily that $\left(I-L_{0}\left(x, \eta^{\prime}, z\right)\right)^{-1}$ has poles only at $\left\{z_{ \pm}^{j}\right\}_{j=1, \ldots, d}$. It follows from Remark 2.4 (ii) in Soga $[6]$ that $\operatorname{dim} \operatorname{Ker}\left[I-L_{0}\left(z_{+}^{j}\right)\right] \leq$ mult $\left[z_{+}^{j}\right]$. Furthermore, we can prove by the same method as for Lemma 2.3 and Remark 2.4 in [6] that the pole at $z_{+}^{j}$ is simple if and only if $\operatorname{dim} \operatorname{Ker}\left[I-L_{0}\left(z_{+}^{j}\right)\right]=\operatorname{mult}\left[z_{+}^{j}\right]$. This equality holds for the real root $z_{+}^{j}$ automatically (cf. the proof of Lemma 2.3 in $[\mathbf{6}]$ ). Therefore, from the 
assumption (A.5) the pole at $z_{+}^{j}$ is always simple. Hence, (i) of Lemma 2.1 is obtained. For (ii) and (iii) of Lemma 2.1, see Lemma 2.5 in Soga [6] and Remark 3.2 in Kawashita, Soga and Ralston $[\mathbf{2}]$ respectively.

The order of the pole of $\left(I-L_{0}\left(x, \eta^{\prime}, z\right)\right)^{-1}$ may increase by differentiating. Because of this, the integral $\int_{c_{+}} e^{i \sigma x_{n}(z+\psi)} g D_{x_{p}}\left(I-L_{0}\left(x, \eta^{\prime}, z\right)\right)^{-1} h \bar{d} z$ may become of higher order in $\sigma$ :

THEOREM 2.2. Let $c\left(z_{+}^{j}\right)$ be a small path surrounding only $z_{+}^{j}$. Assume that $\psi\left(x, \eta^{\prime}\right), g\left(x, \eta^{\prime}, z\right)$ and $h\left(x, \eta^{\prime}\right) \in \mathcal{B}^{\infty}\left(\overline{\mathbb{R}_{+}^{n}} \times \mathbb{R}^{n-1}\right)$ and that $g$ is analytic near $z_{+}^{j}$. Then we have

$$
\begin{aligned}
& \int_{c\left(z_{+}^{j}\right)} e^{i \sigma x_{n}\left(z+\psi\left(x, \eta^{\prime}\right)\right)} g\left(x, \eta^{\prime}, z\right)\left\{D_{x_{p}}\left(I-L_{0}\left(x, \eta^{\prime}, z\right)\right)^{-1}\right\} h\left(x, \eta^{\prime}\right) \bar{d} z \\
& =\int_{c\left(z_{+}^{j}\right)} e^{i \sigma x_{n}(z+\psi)} \sigma x_{n} g\left(\partial_{x_{p}} z_{+}^{j}\right)\left(I-L_{0}(z)\right)^{-1} h \bar{d} z \\
& \quad+\int_{c\left(z_{+}^{j}\right)} e^{i \sigma x_{n}(z+\psi)}\left\{\left(\partial_{z} g\right) R_{0}^{j}\left(D_{x_{p}} L_{0}\right)\left(z_{+}^{j}\right)+g R_{1}^{j}\left(D_{x_{p}} L_{0}\right)\left(z_{+}^{j}\right)\right\}\left(I-L_{0}(z)\right)^{-1} h \bar{d} z \\
& \quad+\int_{c\left(z_{+}^{j}\right)} e^{i \sigma x_{n}(z+\psi)} g\left(I-L_{0}(z)\right)^{-1}\left\{\left(\partial_{z} D_{x_{p}} L_{0}\right)\left(z_{+}^{j}\right)\right) R_{0}^{j} h \\
& \left.\quad+\left(D_{x_{p}} L_{0}\right)\left(z_{+}^{j}\right) R_{1}^{j} h\right\}\left(I-L_{0}(z)\right)^{-1} \bar{d} z
\end{aligned}
$$

where $R_{p}^{j}\left(x, \eta^{\prime}\right)(p=0,1, \ldots)$ are the matrices in (i) of Lemma 2.1. In the above integrals the path $c\left(z_{+}^{j}\right)$ can be changed with $c_{+}$if $g$ is analytic on the area $\left[c_{+}\right]$surrounded by $c_{+}$ and is equal to 0 at $z=z_{+}^{l}$ for every $l \neq j$.

By Theorem 2.2 we obtain immediately the following corollary.

Corollary 2.3. Let $c\left(z_{+}^{j}\right)$ be the path in Theorem 2.2 and let $\psi, g$ and $h$ be the functions in the same theorem. We define the differential operators $B_{x_{p}}^{j}$ and $\tilde{B}_{x_{p}}^{j}$ for $g\left(x, \eta^{\prime}, z\right)$ and $h\left(x, \eta^{\prime}\right)$ respectively by

$$
\begin{aligned}
& B_{x_{p}}^{j} g=D_{x_{p}} g+\left(\partial_{z} g\right) R_{0}^{j} D_{x_{p}} L_{0}\left(z_{+}^{j}\right)+g R_{1}^{j} D_{x_{p}} L_{0}, \\
& \left.\tilde{B}_{x_{p}}^{j} h=D_{x_{p}} h+\left(\partial_{z} D_{x_{p}} L_{0}\right)\left(z_{+}^{j}\right)\right) R_{0}^{j} h+\left(D_{x_{p}} L_{0}\right) R_{1}^{j} h .
\end{aligned}
$$

Then we have

$$
\begin{aligned}
& D_{x_{p}} \int_{c\left(z_{+}^{j}\right)} e^{i \sigma x_{n}\left(z+\psi\left(x, \eta^{\prime}\right)\right)} g\left(x, \eta^{\prime}, z\right)\left(I-L_{0}\left(x, \eta^{\prime}, z\right)\right)^{-1} h\left(x, \eta^{\prime}\right) \bar{d} z \\
& =\int_{c\left(z_{+}^{j}\right)} e^{i \sigma x_{n}(z+\psi)} \sigma\left(\partial_{x_{p}}\left(x_{n} z_{+}^{j}+x_{n} \psi\right)\right) g\left(I-L_{0}(z)\right)^{-1} h \bar{d} z \\
& \quad+\int_{c\left(z_{+}^{j}\right)} e^{i \sigma x_{n}(z+\psi)}\left\{\left(B_{x_{p}}^{j} g\right)\left(I-L_{0}(z)\right)^{-1} h+g\left(I-L_{0}(z)\right)^{-1} \tilde{B}_{x_{p}}^{j} h\right\} \bar{d} z .
\end{aligned}
$$

In the above integrals the path $c\left(z_{+}^{j}\right)$ can be changed with $c_{+}$if $g$ is analytic on $\left[c_{+}\right]$and 
$g(z), B_{x_{p}}^{l} g(z)$ are equal to 0 at $z=z_{+}^{l}$ for every $l \neq j$.

Proof of Theorem 2.2. Noting that $D_{x_{p}}\left(I-L_{0}\left(x, \eta^{\prime}, z\right)\right)^{-1}=\left(I-L_{0}(z)\right)^{-1}$ $\left(D_{x_{p}} L_{0}(z)\right)\left(I-L_{0}(z)\right)^{-1}$, by the expansion in (i) of Lemma 2.1 we have

$$
\begin{aligned}
& D_{x_{p}}\left(I-L_{0}(z)\right)^{-1}=\left(z-z_{+}^{j}\right)^{-2} R_{0}^{j}\left(D_{x_{p}} L_{0}\right)\left(z_{+}^{j}\right) R_{0}^{j} \\
&+\left(z-z_{+}^{j}\right)^{-1}\left\{R_{0}^{j}\left(D_{x_{p}} L_{0}\right)\left(z_{+}^{j}\right) R_{1}^{j}+R_{1}^{j}\left(D_{x_{p}} L_{0}\right)\left(z_{+}^{j}\right) R_{0}^{j}\right\}+\cdots \\
& \text { at } z=z_{+}^{j} .
\end{aligned}
$$

This yields that

$$
\begin{aligned}
& \int_{c\left(z_{+}^{j}\right)} e^{i \sigma x_{n}(z+\psi)} g(z)\left\{D_{x_{p}}\left(I-L_{0}(z)\right)^{-1}\right\} h \bar{d} z \\
& =\int_{c\left(z_{+}^{j}\right)} e^{i \sigma x_{n}(z+\psi)} i \sigma x_{n} g\left(I-L_{0}(z)\right)^{-1}\left(D_{x_{p}} L_{0}\right) R_{0}^{j} h \bar{d} z \\
& \quad+\int_{c\left(z_{+}^{j}\right)} e^{i \sigma x_{n}(z+\psi)}\left(\left(\partial_{z} g\right) R_{0}^{j}\left(D_{x_{p}} L_{0}\right)+g R_{1}^{j}\left(D_{x_{p}} L_{0}\right)\right)\left(I-L_{0}(z)\right)^{-1} h \bar{d} z \\
& \quad+\int_{c\left(z_{+}^{j}\right)} e^{i \sigma x_{n}(z+\psi)} g\left(I-L_{0}(z)\right)^{-1}\left(\left(\partial_{z} D_{x_{p}} L_{0}\right) R_{0}^{j}+\left(D_{x_{p}} L_{0}\right) R_{1}^{j}\right) h \bar{d} z .
\end{aligned}
$$

Therefore, we obtain Theorem 2.2 if the leading terms of the right hands in (2.1) and (2.2) coincide each other, i.e.,

$$
\begin{aligned}
& \int_{c\left(z_{+}^{j}\right)} e^{i \sigma x_{n}(z+\psi)} i \sigma x_{n} g\left(I-L_{0}(z)\right)^{-1}\left(D_{x_{p}} L_{0}\right) R_{0}^{j} h \overline{d z} \\
& =\int_{c\left(z_{+}^{j}\right)} e^{i \sigma x_{n}(z+\psi)} i \sigma x_{n} g\left(\partial_{x_{p}} z_{+}^{j}\right)\left(I-L_{0}(z)\right)^{-1} h \overline{d z} .
\end{aligned}
$$

Calculating the residues of the integrals, we see from (i) of Lemma 2.1 that the above equality means

$$
e^{i \sigma x_{n}\left(z_{+}^{j}+\psi\right)} i \sigma x_{n} g\left(z_{+}^{j}\right) R_{0}^{j}\left(D_{x_{p}} L_{0}\right) R_{0}^{j} h=e^{i \sigma x_{n}\left(z_{+}^{j}+\psi\right)} i \sigma x_{n} g\left(z_{+}^{j}\right) \partial_{x_{p}} z_{+}^{j} R_{0}^{j} h .
$$

Applying $D_{x_{p}}$ to the expansion in (i) of Lemma 2.1, we have

$$
D_{x_{p}}\left(I-L_{0}(z)\right)^{-1}=\frac{D_{x_{p}} z_{+}^{j}}{\left(z-z_{+}^{j}\right)^{2}} R_{0}^{j}+\frac{1}{z-z_{+}^{j}} D_{x_{p}} R_{0}^{j}+\cdots .
$$

Comparing the terms of the order $\left(z-z_{+}^{j}\right)^{-2}$ in this expansion and (2.2), we have

$$
R_{0}^{j}\left(D_{x_{p}} L_{0}\right) R_{0}^{j}=\partial_{x_{p}} z_{+}^{j} R_{0}^{j} .
$$

Therefore, we get the equality (2.4) and obtain Theorem 2.2. The proof is complete.

It seems that the first term in (2.1) is associated with the principal part. However, 
the term for the non-real root $z_{+}^{j}(j=k+1, \ldots, d)$ becomes of lower order. This is seen from (ii) of the following lemma.

LEMma 2.4. Let $\psi\left(x, \eta^{\prime}\right), g\left(x, \eta^{\prime}, z\right)$ and $h\left(x, \eta^{\prime}\right) \in \mathcal{B}^{\infty}\left(\overline{\mathbb{R}_{+}^{n}} \times \mathbb{R}^{n-1}\right)$ and $g$ be analytic on $\left[c_{+}\right]$(the area surrounded by $c_{+}$). Assume that $z+\psi$ is real-valued or $\operatorname{Im}(z+\psi) \geq \delta$ $\left(x_{n} \in J\right)$ with a constant $\delta>0$ at each pole of $g(z)\left(I-L_{0}(z)\right)^{-1}$. Then we obtain the following (i)-(iii) $\left(x_{n} \in J\right)$.

(i) $\mathrm{Op}\left[\int_{c_{+}} e^{i \sigma x_{n}(z+\psi)} g(z)\left(I-L_{0}(z)\right)^{-1} h \bar{d} z\right]$ is a bounded operator from $H^{s}$ to $\mathcal{H}^{s, s}$ for any non-negative integer $s$.

(ii) Let $c_{+}^{I}$ be a small path surrounding only all the poles with $\operatorname{Im}(z+\psi) \geq \delta$. Then we obtain for any positive integer $m$

$$
\left|\int_{c_{+}^{I}} e^{i \sigma x_{n}(z+\psi)} x_{n}^{m} g(z)\left(I-L_{0}(z)\right)^{-1} h \bar{d} z\right| \leq C_{m}(\sigma+1)^{-m} \quad(\sigma>0) .
$$

(iii) Let $c_{+}^{I}$ be the path in the above (ii). Then, $\mathrm{Op}\left[\int_{c_{+}^{I}} e^{i \sigma x_{n}(z+\psi)} x_{n}^{m} g(z)\left(I-L_{0}(z)\right)^{-1}\right.$ $h \overline{d z}]$ is a bounded operator from $H^{s}$ to $\mathcal{H}^{s, s+m}$. Here, the path $c_{+}^{I}$ can be changed with $c_{+}$if $g$ is analytic on $\left[c_{+}\right]$and equal to 0 at all the poles outside $\left[c_{+}^{I}\right]$.

Proof. At all the poles in $\left[c_{+}^{I}\right]$ we have

$$
\left|e^{i \sigma x_{n}(z+\psi)} x_{n}^{m} \sigma^{m}\right| \leq\left(\sigma x_{n}\right)^{m} e^{-\delta \sigma x_{n}} \leq C_{m} .
$$

(ii) of the lemma follows from this inequality.

Let $S_{1,0}^{\tilde{m}}$ be the symbol class with the weight function $<\sigma, \xi^{\prime}>=\left(\sigma^{2}+\left|\xi^{\prime}\right|^{2}\right)^{1 / 2}$ (cf. Section 1 in Chapter 2 of Kumano-go [3]); i.e. ' $p\left(x^{\prime}, \sigma, \xi^{\prime}\right) \in S_{1,0}^{\tilde{m}}$ ' means that for any $\alpha$ and $\beta$

$$
\left|\partial_{x^{\prime}}^{\alpha} \partial_{\left(\sigma, \xi^{\prime}\right)}^{\beta} p\left(x^{\prime}, \sigma, \xi^{\prime}\right)\right| \leq C_{\alpha, \beta}<\sigma, \xi^{\prime}>^{\tilde{m}-|\beta|} .
$$

For $p\left(x^{\prime}, \sigma, \xi^{\prime}\right) \in S_{1,0}^{\tilde{m}}$ we define the pseudo-differential operator by $p\left(x^{\prime}, D_{t}, D_{x^{\prime}}\right) f=$ $\iint e^{i t \sigma+i x^{\prime} \xi^{\prime}} p\left(x^{\prime}, \sigma, \xi^{\prime}\right) \hat{f}\left(\sigma, \xi^{\prime}\right) \bar{d} \sigma \bar{d} \xi^{\prime}$. If $p\left(x^{\prime}, \sigma, \xi^{\prime}\right) \in S_{1,0}^{\tilde{m}}$, we have

$$
\left\|p\left(x^{\prime}, D_{t}, D_{x^{\prime}}\right) f\right\|_{H^{s}} \leq C_{s}\|f\|_{H^{\tilde{m}+s}}
$$

(cf. Theorem 2.7 in Chapter 3 of Kumano-go [3]). We set

$$
\begin{aligned}
& q\left(x^{\prime}, x_{n}, \sigma, \xi^{\prime}\right) \\
& \quad=\int_{c_{+}^{I}} e^{i \sigma x_{n}\left(z+\psi\left(x, \xi^{\prime} / \sigma\right)\right)} x_{n}^{m} g\left(x, \xi^{\prime} / \sigma, z\right)\left(I-L_{0}\left(x, \xi^{\prime} / \sigma, z\right)\right)^{-1} h\left(x, \xi^{\prime} / \sigma\right) d z \chi\left(x, \sigma, \xi^{\prime} / \sigma\right),
\end{aligned}
$$

where we take the cutoff function $\chi$ so that $\chi\left(x, \sigma, \eta^{\prime}\right)=0$ for $\sigma<1 / 2$. Then, taking the residues in $\left[c_{+}^{I}\right]$, we see from (ii) of Lemma 2.4 that $\partial_{x_{n}}^{l} q\left(x, \sigma, \xi^{\prime}\right)$ belongs to $C^{0}\left(J ; S_{1,0}^{-m+l}\right)(l=0,1, \ldots, s)$ since the inequality $C^{-1}<\sigma, \xi^{\prime}>^{\tilde{m}} \leq \sigma^{\tilde{m}} \leq C<\sigma, \xi^{\prime}>^{\tilde{m}}$ holds for $\left(\sigma, \xi^{\prime}\right)$ satisfying $\chi\left(x, \sigma, \xi^{\prime} / \sigma\right) \neq 0$. Therefore, using (2.6), we have 


$$
\left\|\partial_{x_{n}}^{l} q\left(x^{\prime}, x_{n}, D_{t}, D_{x^{\prime}}\right) f\right\|_{H^{s+m-l}} \leq C\|f\|_{H^{s}} .
$$

Furthermore, $\partial_{x_{n}}^{l} \mathrm{Op}\left[\int_{c_{+}^{I}} e^{i \sigma x_{n}(z+\psi)} x_{n}^{m} g(z)\left(I-L_{0}(z)\right)^{-1} h \bar{d} z\right] f$ is equal to $\partial_{x_{n}}^{l} q\left(x^{\prime}, x_{n}, D_{t}\right.$, $\left.D_{x^{\prime}}\right) f$. Hence, we obtain (iii) of Lemma 2.4 when the integral is of the form $\int_{c_{+}^{I}} \cdots \bar{d} z$. If $g(z)=0$ at all the poles outside $\left[c_{+}^{I}\right]$, it is obvious that $\int_{c_{+}^{I}} e^{i \sigma x_{n}(z+\psi)} x_{n}^{m} g(z)(I-$ $\left.L_{0}(z)\right)^{-1} h \bar{d} z=\int_{c_{+}} e^{i \sigma x_{n}(z+\psi)} x_{n}^{m} g(z)\left(I-L_{0}(z)\right)^{-1} h \bar{d} z$. Therefore all the statements in (iii) are obtained.

Let us verify (i) of Lemma 2.4. We have $\mathrm{Op}\left[\int_{c_{+}} e^{i \sigma x_{n}(z+\psi)} g(z)\left(I-L_{0}(z)\right)^{-1} h \overline{d z}\right]=$ $\mathrm{Op}\left[\int_{c_{+}^{I}} e^{i \sigma x_{n}(z+\psi)} g(z)\left(I-L_{0}(z)\right)^{-1} h \bar{d} z\right]+\sum_{j=1}^{\tilde{k}} \mathrm{Op}\left[e^{i \sigma x_{n}\left(\tilde{z}^{j}+\psi\right)} g\left(\tilde{z}^{j}\right) \tilde{R}^{j} h\right]$, where $\tilde{z}^{j}$ are the poles outside $\left[c_{+}^{I}\right]$ and $\tilde{R}^{j}=\operatorname{Res}_{z=z^{j}}\left(I-L_{0}(z)\right)^{-1}$. We have shown that $\mathrm{Op}\left[\int_{c_{+}^{I}} e^{i \sigma x_{n}(z+\psi)} g(z)\left(I-L_{0}(z)\right)^{-1} h \bar{d} z\right]$ is bounded from $H^{s}$ to $\mathcal{H}^{s, s}$. Op $\left[e^{i \sigma x_{n}\left(\tilde{z}^{j}+\psi\right)}\right.$ $\left.g\left(\tilde{z}^{j}\right) \tilde{R}^{j} h\right] f$ is of the form

$$
\iint e^{i t \sigma+i x^{\prime} \xi^{\prime}+i \sigma x_{n}\left(\tilde{z}^{j}+\psi\left(x, \xi^{\prime} / \sigma\right)\right)} g\left(\tilde{z}^{j}\right) \tilde{R}^{j} h \chi\left(x, \sigma, \xi^{\prime} / \sigma\right) \hat{f}\left(\sigma, \xi^{\prime}\right) \bar{d} \sigma \bar{d} \xi^{\prime} .
$$

This is a Fourier integral operator on $\mathbb{R}_{t} \times \mathbb{R}_{x^{\prime}}^{n-1}$ with the phase function (with the parameter $x_{n} \in J$ )

$$
\phi\left(t, x, \sigma, \xi^{\prime}\right)=t \sigma+x^{\prime} \xi^{\prime}+\sigma x_{n}\left(\tilde{z}^{j}\left(x, \xi^{\prime} / \sigma\right)+\psi\left(x, \xi^{\prime} / \sigma\right)\right),
$$

and the symbol $g\left(\tilde{z}^{j}\right) \tilde{R}^{j} h \chi$ belongs to $S_{1,0}^{0}$. We can check that the function $\phi\left(t, x, \sigma, \eta^{\prime}\right)$ satisfies the conditions for the real-valued phase functions stated in Kumano-go [3] (cf. Definition 1.2 in Chapter 10) if the interval $J$ is small enough. Therefore, by Theorem 2.3 in Chapter 10 of $[\mathbf{3}]$, we obtain

$$
\left\|\mathrm{Op}\left[e^{i \sigma x_{n}\left(\tilde{z}^{j}+\psi\right)} g\left(\tilde{z}^{j}\right) \tilde{R}^{j} h\right] f\right\|_{H^{s}} \leq C\|f\|_{H^{s}} .
$$

We can confirm the similar property for $\partial_{x_{n}}^{l} \mathrm{Op}\left[e^{i \sigma x_{n}\left(\tilde{z}^{j}+\psi\right)} g\left(\tilde{z}^{j}\right) \tilde{R}^{j} h\right]$ also. Thus (i) is proved. The proof is complete.

The principal part of the asymptotic solution in Theorem 1 can be made by superposing the forms $\mathrm{Op}\left[\int_{c_{+}} e^{i \sigma x_{n}\left(z+\varphi^{j}\right)} \tilde{g}_{0}^{j}\left(I-L_{0}(z)\right)^{-1} \tilde{h}_{0}^{j} d z\right] f(j=1, \ldots, d)$. This proof is based on Theorem 2 in Introduction (Section 1). Theorem 2 follows from the following theorem.

Theorem 2.5. Let $\left(\tilde{x}^{\prime}, \tilde{\eta}^{\prime}\right)$ be non-glancing. Then in a neighborhood $U$ of $\left(\tilde{x}^{\prime}, 0, \tilde{\eta}^{\prime}\right)$ we have

$$
\sum_{j=1}^{d}\left\{\operatorname{Res}_{z=z_{+}^{j}}\left(I-L_{0}\left(x, \eta^{\prime}, z\right)\right)^{-1}\right\} \mathbb{C}^{n}=\mathbb{C}^{n} \quad \text { for }\left(x, \eta^{\prime}\right) \in U,
$$

i.e., for any $v \in \mathbb{C}^{n}$ there exist $v_{j} \in\left\{\operatorname{Res}_{z=z_{+}^{j}}\left(I-L_{0}\left(x, \eta^{\prime}, z\right)\right)^{-1}\right\} \mathbb{C}^{n}$ such that $v=$ $\sum_{j=1}^{d} v_{j}$ 
Let us note that this theorem is equivalent to (1.5) (cf. (ii) of Lemma 2.1 also).

Proof of Theorem 2.5. Let $v \in \mathbb{C}^{n}$ be orthogonal to $\sum_{j=1}^{d}\left\{\operatorname{Res}_{z=z_{+}^{j}}(I-\right.$ $\left.\left.L_{0}\left(x, \eta^{\prime}, z\right)\right)^{-1}\right\} \mathbb{C}^{n}$. To prove Theorem 2.5 , we have only to show that $v=0$. Denote the residues of $\left(I-L_{0}(z)\right)^{-1}$ at $z=z_{ \pm}^{j}$ by $R_{ \pm}^{j}(j=1, \ldots, d)$. Note that $R_{+}^{j}=R_{0}^{j}$ $(j=1, \ldots, d)$ and that $R_{ \pm}^{j}=\left(\partial_{\xi_{n}} \lambda_{j}\left(z_{ \pm}^{j}\right)\right)^{-1} P_{ \pm}^{j}(j=1, \ldots, k)$, where $P_{ \pm}^{j}$ are the orthogonal projections to the eigenspaces of the eigenvalues $\lambda_{j}\left(z_{ \pm}^{j}\right)$ (cf. (iii) in Lemma 2.1). By calculation of the residue at $z=\infty$, for large $r>0$ we have

$$
\begin{aligned}
\sum_{j=1}^{d}\left(z_{+}^{j}\right)^{l} R_{+}^{j}+\sum_{j=1}^{d}\left(z_{-}^{j}\right)^{l} R_{-}^{j} & =\int_{|z|=r} z^{l}\left(I-L_{0}(z)\right)^{-1} \bar{d} z \\
& =0 \quad(\text { when } l=0), \quad=-a_{n n}^{-1} \quad(\text { when } l=1),
\end{aligned}
$$

where $a_{n n}$ is the coefficient of $D_{x_{n}}^{2}$ in $L_{0}\left(x, D_{x}\right)$.

Since ${ }^{t} \overline{\left(I-L_{0}(x, z)\right)^{-1}}=\left(I-L_{0}(x, \bar{z})\right)^{-1}$ for $j=k+1, \ldots, d$, it follows that ${ }^{t} \overline{R_{-}^{j}}=$ $-R_{+}^{j}$ for $j=k+1, \ldots, d$. Hence, noting that $v$ is orthogonal to $R_{+}^{j} \mathbb{C}^{n}$ for $j=1, \ldots, d$, we have $\left(R_{+}^{j} v, v\right)=0$ for $j=1, \ldots, d$ and $\left(R_{-}^{j} v, v\right)=\left(v,{ }^{t} \overline{R_{-}^{j}} v\right)=0$ for $j=k+1, \ldots, d$. Combining this and (2.7), we get

$$
\sum_{j=1}^{k}\left(R_{-}^{j} v, v\right)=0, \quad \sum_{j=1}^{k} z_{-}^{j}\left(R_{-}^{j} v, v\right)=-\left(a_{n n}^{-1} v, v\right) .
$$

The first equality in (2.8) means that $\sum_{j=1}^{k}\left(\partial_{\xi_{n}} \lambda_{j}\left(z_{-}^{j}\right)\right)^{-1}\left(P_{-}^{j} v, v\right)=0$. All the terms in this sum are non-negative since $\partial_{\xi_{n}} \lambda_{j}\left(z_{-}^{j}\right)>0(j=1, \ldots, k)$. Hence, $\left(R_{-}^{j} v, v\right)=0$ holds for $j=1, \ldots, k$. Therefore, using the second equality in $(2.8)$, we have $\left(a_{n n}^{-1} v, v\right)=0$, which yields $v=0$. Thus the theorem is proved.

At the end of this section we prove Theorem 2 in Introduction (Section 1).

Proof of Theorem 2. Let $n^{j}$ be the dimension of $R_{+}^{j}\left(\tilde{x}^{\prime}, 0, \tilde{\eta}^{\prime}\right) \mathbb{C}^{n}$ and take $e_{l}^{j} \in \mathbb{C}^{n}\left(l=1, \ldots, n^{j}\right)$ such that $\left\{R_{+}^{j}\left(\tilde{x}^{\prime}, 0, \tilde{\eta}^{\prime}\right) e_{l}^{j}\right\}_{l=1, \ldots, n^{j}}$ are bases in $R_{+}^{j}\left(\tilde{x}^{\prime}, 0, \tilde{\eta}^{\prime}\right) \mathbb{C}^{n}$. Then, Theorem 2.5 means that $\left\{R_{+}^{j}\left(\tilde{x}^{\prime}, 0, \tilde{\eta}^{\prime}\right) e_{l}^{j} \mid j=1, \ldots, d, l=1, \ldots, n^{j}\right\}$ expands $\mathbb{C}^{n}$. Therefore, if the number of $\left\{R_{+}^{j} e_{l}^{j}\right\}$ is equal to $n$ (i.e. $n=\sum_{j=1}^{d} n^{j}$ ), $\left\{R_{+}^{j} e_{l}^{j}\right\}$ are linearly independent. For the real roots $z_{+}^{j}$ (i.e. $j=1, \ldots, k$ ) we have $n^{j}=\operatorname{mult}\left[z_{+}^{j}\right]$ (cf. Remark 2.4 in $[6]$ ), and mult $\left[z_{+}^{j}\right]=\operatorname{mult}\left[z_{-}^{j}\right]$ by (A.4). Furthermore, the same equalities for $j=k+1, \ldots, d$ follow from (A.5). Therefore, noting that $\sum_{j=1}^{d} \operatorname{mult}\left[z_{+}^{j}\right]+\sum_{j=1}^{d} \operatorname{mult}\left[z_{-}^{j}\right]=2 n$, we have $\sum_{j=1}^{d} n^{j}=n$. Hence, $H\left(x, \eta^{\prime}\right)=\left(R_{+}^{1}\left(x, \eta^{\prime}\right) e_{1}^{1}, \cdots, R_{+}^{1}\left(x, \eta^{\prime}\right) e_{n^{1}}^{1}, \cdots, R_{+}^{d}\left(x, \eta^{\prime}\right) e_{1}^{d}, \cdots, R_{+}^{d}\left(x, \eta^{\prime}\right) e_{n^{d}}^{d}\right)$ is an $n \times n$ matrix and $\left\{R_{+}^{l}\left(\tilde{x}^{\prime}, 0, \tilde{\eta}^{\prime}\right) e_{l}^{j}\right\}$ are linearly independent, which yields that $\operatorname{det} H\left(x, \eta^{\prime}\right) \neq 0$ for any $\left(x, \eta^{\prime}\right) \in U$ (if $U$ is small enough).

$H\left(x, \eta^{\prime}\right)$ is rewritten in the form $H\left(x, \eta^{\prime}\right)=\sum_{j=1}^{d} R_{+}^{j}\left(x, \eta^{\prime}\right)\left(0, \cdots, e_{1}^{j}, \cdots, e_{n^{j}}^{j}\right.$, $0, \cdots, 0)$. Multiply this equality by $H\left(x, \eta^{\prime}\right)^{-1}$ from right hand. And set $G^{j}\left(x, \eta^{\prime}\right)=$ $\left(0, \cdots, e_{1}^{j}, \cdots, e_{n^{j}}^{j}, 0, \cdots, 0\right) H\left(x, \eta^{\prime}\right)^{-1}$. Then we have 


$$
\sum_{j=1}^{d} R_{+}^{j}\left(x, \eta^{\prime}\right) G^{j}\left(x, \eta^{\prime}\right)=I
$$

This yields that $\sum_{j=1}^{d}{ }^{t} G^{j}\left(x, \eta^{\prime}\right) R_{+}^{j}\left(x, \eta^{\prime}\right)=I$ since $R_{+}^{j}\left(x, \eta^{\prime}\right)$ is symmetric. Thus Theorem 2 is obtained.

\section{Basic examination for the construction of the solutions.}

In this section we examine the forms of $\mathrm{Op}\left[\int_{c_{+}} e^{i \sigma x_{n}(z+\psi)} g(z)\left(I-L_{0}(z)\right)^{-1} h \bar{d} z\right]$ for differentiation in $x_{j}$, where $g(z)$ is analytic on $\left[c_{+}\right]$(the area surrounded by $c_{+}$). Let $\left(x, \eta^{\prime}\right)$ move in an open set $U=U_{\left(x^{\prime}, \eta^{\prime}\right)}^{\prime} \times J_{x_{n}}$.

For a while we restrict the path $c_{+}$in $\mathrm{Op}\left[\int_{c_{+}} \cdots \bar{d} z\right]$ to a small path $c\left(z_{+}^{l}\right)$ surrounding only the root $z_{+}^{l}(l=1, \ldots, d)$.

TheOREM 3.1. Let $g\left(x, \eta^{\prime}, z\right)$ and $h\left(x, \eta^{\prime}\right)$ be $n \times n$ matrices $\left(\in \mathcal{B}^{\infty}(\bar{U})\right)$ and $g$ be analytic in $z$ near $z=z_{+}^{l}$. Let $\psi\left(x, \eta^{\prime}\right)$ be a scalar-valued function $\left(\in \mathcal{B}^{\infty}(\bar{U})\right)$. For $p=1, \ldots, n$ we set

$$
a_{p}=\sum_{q=1}^{n}\left(a_{p q}+a_{q p}\right)\left(\eta_{q}+x_{n} \partial_{x_{q}}\left(z_{+}^{l}+\psi\right)\right), \quad \eta_{n}=z_{+}^{l}+\psi .
$$

Let $B_{x_{p}}^{l}$ and $\tilde{B}_{x_{p}}^{l}$ be the operators in Corollary 2.3. Then we have

$$
\begin{aligned}
& \left(D_{t}^{2}-L\left(x, D_{x}\right)\right) \operatorname{Op}\left[\int_{c\left(z_{+}^{l}\right)} e^{i \sigma x_{n}(z+\psi)} g\left(x, \eta^{\prime}, z\right)\left(I-L_{0}\left(x, \eta^{\prime}, z\right)\right)^{-1} h\left(x, \eta^{\prime}\right) \bar{d} z\right] f \\
& =\operatorname{Op}\left[\int_{c\left(z_{+}^{l}\right)} e^{i \sigma x_{n}(z+\psi)}\left\{I-L_{0}\left(x, \eta+x_{n} \partial_{x}\left(z_{+}^{l}+\psi\right)\right)\right\} g\left(I-L_{0}\left(x, \eta^{\prime}, z\right)\right)^{-1} h \bar{d} z \sigma^{2}\right] f \\
& \quad-\operatorname{Op}\left[\int_{c\left(z_{+}^{l}\right)} e^{i \sigma x_{n}(z+\psi)} \sum_{p=1}^{n} a_{p}\left\{g\left(I-L_{0}(z)\right)^{-1} \tilde{B}_{x_{p}}^{l} h+\left(B_{x_{p}}^{l} g\right)\left(I-L_{0}(z)\right)^{-1} h\right\} \bar{d} z \sigma\right] f \\
& -\operatorname{Op}\left[\int_{c\left(z_{+}^{l}\right)} e^{i \sigma x_{n}(z+\psi)} L\left\langle g(z)\left(I-L_{0}(z)\right)^{-1} h\right\rangle \bar{d} z\right] f,
\end{aligned}
$$

where $L\left\langle g\left(I-L_{0}(z)\right)^{-1} h\right\rangle=\sum_{p, q=1}^{n} a_{p q}\left\{\left(B_{x_{p}}^{l} B_{x_{q}}^{l} g\right)\left(I-L_{0}\right)^{-1} h+\left(B_{x_{p}}^{l} g\right)\left(I-L_{0}\right)^{-1} \tilde{B}_{x_{q}}^{l} h+\right.$ $\left.\left(B_{x_{q}}^{l} g\right)\left(I-L_{0}\right)^{-1} \tilde{B}_{x_{p}}^{l} h+g\left(I-L_{0}\right)^{-1} \tilde{B}_{x_{p}}^{l} \tilde{B}_{x_{q}}^{l} h\right\}+\sum_{p=1}^{n} b_{p}\left\{\left(B_{x_{p}}^{l} g\right)\left(I-L_{0}\right)^{-1} h+g(I-\right.$ $\left.\left.L_{0}\right)^{-1} \tilde{B}_{x_{p}}^{l} h\right\}+b_{0} g\left(I-L_{0}\right)^{-1} h$.

Proof. By Corollary 2.3 we have

$$
\begin{aligned}
& D_{x_{p}} D_{x_{q}} \int_{c\left(z_{+}^{l}\right)} e^{i \sigma x_{n}(z+\psi)} g(z)\left(I-L_{0}(z)\right)^{-1} h \bar{d} z \\
& \quad=\int_{c\left(z_{+}^{l}\right)} e^{i \sigma x_{n}(z+\psi)} \sigma^{2}\left\{\partial_{x_{p}}\left(x_{n} z_{+}^{l}+x_{n} \psi\right) \partial_{x_{q}}\left(x_{n} z_{+}^{l}+x_{n} \psi\right)\right\} g(z)\left(I-L_{0}(z)\right)^{-1} h \bar{d} z
\end{aligned}
$$




$$
\begin{gathered}
+\int_{c\left(z_{+}^{l}\right)} e^{i \sigma x_{n}(z+\psi)} \sigma\left\{\partial_{x_{p}}\left(x_{n} z_{+}^{l}+x_{n} \psi\right) B_{x_{q}} g+\partial_{x_{q}}\left(x_{n} z_{+}^{l}+x_{n} \psi\right) B_{x_{p}} g\right. \\
\left.+\left(D_{x_{p}} \partial_{x_{q}}\left(x_{n} z_{+}^{l}+x_{n} \psi\right)\right) g\right\}\left(I-L_{0}(z)\right)^{-1} h \overline{d z} \\
+\int_{c\left(z_{+}^{l}\right)} e^{i \sigma x_{n}(z+\psi)} \sigma g\left(I-L_{0}(z)\right)^{-1}\left\{\partial_{x_{p}}\left(x_{n} z_{+}^{l}+x_{n} \psi\right) \tilde{B}_{x_{q}} h\right. \\
\left.+\partial_{x_{q}}\left(x_{n} z_{+}^{l}+x_{n} \psi\right) \tilde{B}_{x_{p}} h\right\} d z \\
+\int_{c\left(z_{+}^{l}\right)} e^{i \sigma x_{n}(z+\psi)}\left\{\left(B_{x_{p}} B_{x_{q}} g\right)\left(I-L_{0}(z)\right)^{-1} h+\left(B_{x_{p}} g\right)\left(I-L_{0}(z)\right)^{-1} \tilde{B}_{x_{q}} h\right. \\
\left.+\left(B_{x_{q}} g\right)\left(I-L_{0}(z)\right)^{-1} \tilde{B}_{x_{p}} h+g\left(I-L_{0}(z)\right)^{-1} \tilde{B}_{x_{p}} \tilde{B}_{x_{q}} h\right\} d z .
\end{gathered}
$$

$\left(D_{t}^{2}-L\right) \operatorname{Op}\left[\int e^{i \sigma x_{n}(z+\psi)} \cdots \bar{d} z\right]$ is of the form $\mathrm{Op}\left[\sigma^{2} \int e^{i \sigma x_{n}(z+\psi)} \cdots \bar{d} z\right]-\sum_{p, q=1}^{n} a_{p q}$ $\operatorname{Op}\left[\left(\tilde{\eta}_{p}+D_{x_{p}}\right)\left(\tilde{\eta}_{q}+D_{x_{q}}\right) \int e^{i \sigma x_{n}(z+\psi)} \cdots \overline{d z}\right]-\sum_{p=1}^{n} b_{p} \operatorname{Op}\left[\left(\tilde{\eta}_{p}+D_{x_{p}}\right) \int e^{i \sigma x_{n}(z+\psi)} \cdots \overline{d z}\right]-$ $b_{0} \mathrm{Op}\left[\int e^{i \sigma x_{n}(z+\psi)} \ldots \bar{d} z\right]$, where $\tilde{\eta}_{j}=\eta_{j}$ for $j=1, \ldots, n-1$ and $\tilde{\eta}_{n}=0$.

Combining this with (3.1) and Corollary 2.3, we see that when applying $\left(D_{t}^{2}-L\right)$ to $\mathrm{Op}\left[\int_{c\left(z_{+}^{l}\right)} e^{i \sigma x_{n}(z+\psi)} g(z)\left(I-L_{0}(z)\right)^{-1} h \bar{d} z\right] f$, the terms with the order $\sigma^{2}$ are of the form $\mathrm{Op}\left[\int_{c\left(z_{+}^{l}\right)} e^{i \sigma x_{n}(z+\psi)} \sigma^{2}\left\{I-L_{0}\left(x, \eta+x_{n} \partial_{x}\left(z_{+}^{l}+\psi\right)\right)\right\} g(z)\left(I-L_{0}\left(x, \eta^{\prime}, z\right)\right)^{-1} h d z\right] f$, where $\eta=\left(\eta^{\prime}, z_{+}^{l}+\psi\right)$. Furthermore, selecting the terms with the order $\sigma^{1}$, we can assemble them into the form $\mathrm{Op}\left[\sum_{q=1}^{n} \int_{c\left(z_{+}^{l}\right)} e^{i \sigma x_{n}(z+\psi)} \sigma\left(a_{p q}+a_{q p}\right)\left(\eta_{q}+x_{n} \partial_{x_{q}}\left(z_{+}^{l}+\psi\right)\right)\left\{B_{x_{p}} g(I-\right.\right.$ $\left.\left.\left.L_{0}(z)\right)^{-1} h+g\left(I-L_{0}(z)\right)^{-1} \tilde{B}_{x_{p}} h+\left(D_{x_{p}} \partial_{x_{q}}\left(x_{n} z_{+}^{l}+x_{n} \psi\right)\right) g\left(I-L_{0}(z)\right)^{-1} h\right\} \bar{d} z\right] f$. The terms with the order $\sigma^{0}$ can also be done similarly. Thus Theorem 3.1 is obtained.

We employ the function $\rho^{j}\left(x, \eta^{\prime}, z\right)=\prod_{l \neq j}\left(z_{+}^{j}-z_{+}^{l}\right)^{-3}\left(z-z_{+}^{l}\right)^{3} \cdot \rho^{j}(z)$ is analytic on $\left[c_{+}\right]$and satisfies

$$
\rho^{j}\left(x, \eta^{\prime}, z_{+}^{j}\right)=1, \quad \partial_{(x, z)}^{\alpha} \rho^{j}\left(x, \eta^{\prime}, z_{+}^{l}\right)=0 \quad \text { for every } l \neq j \text { and }|\alpha| \leq 2 .
$$

Let us note that if $g(z)$ is of the form $\rho^{j}(z) \tilde{g}(z)$ for an analytic function $\tilde{g}(z)$ near $\left[c_{+}\right]$, we have $\partial_{x}^{\alpha} \int_{c_{+}} e^{i \sigma x_{n}(z+\psi)} g(z)\left(I-L_{0}(z)\right)^{-1} h d z=\partial_{x}^{\alpha} \int_{c\left(z_{+}^{j}\right)} e^{i \sigma x_{n}(z+\psi)} g(z)(I-$ $\left.L_{0}(z)\right)^{-1} h \bar{d} z(|\alpha| \leq 2)$. Furthermore, if $g(z)=\rho^{j}(z) \tilde{g}(z)$ and $\operatorname{Im}\left(z_{+}^{j}+\psi\right) \geq \delta(>0)$, $\mathrm{Op}\left[\int_{c_{+}} e^{i \sigma x_{n}(z+\psi)} x_{n}^{m} g(z)\left(I-L_{0}(z)\right)^{-1} h \bar{d} z\right]$ is a bounded operator from $H^{s}$ to $\mathcal{H}^{s, s+m}$ $(s=0,1,2, \ldots)$ (cf. (iii) of Lemma 2.4).

For $j, l=1, \ldots, d$ we set

$$
\psi^{j l}\left(x, \eta^{\prime}\right)=z_{+}^{j}\left(x, \eta^{\prime}\right)+\varphi^{j}\left(x, \eta^{\prime}\right)-z_{+}^{l}\left(x, \eta^{\prime}\right)
$$

where $\varphi^{j}$ is a $C^{\infty}$ function with $\left.\varphi^{j}\right|_{x_{n}=0}=0$ (determined later). Let us note that $\left.\left(z+\psi^{j l}\right)\right|_{z=z_{+}^{l}}$ is equal to $z_{+}^{j}+\varphi^{j}$ and independent of $l$. Furthermore, $a_{p}$ in Theorem 3.1 also does not depend on $l$ if $\psi=\psi^{j l}$. Let $M^{l}$ be the matrix in Theorem 2 and set

$$
g^{l}\left(x, \eta^{\prime}, z\right)=\rho^{l}\left(x, \eta^{\prime}, z\right) M^{l}\left(x, \eta^{\prime}\right), \quad l=1, \ldots, d .
$$


Applying Theorem 3.1 with $\psi=\psi^{j l}$ and $g=g^{l}$, we obtain the following corollary since $\left(D_{t}^{2}-L\right) \operatorname{Op}\left[\int_{c_{+}} e^{i \sigma x_{n}\left(z+\psi^{j l}\right)} g^{l}\left(I-L_{0}(z)\right)^{-1} h \bar{d} z\right]=\left(D_{t}^{2}-L\right) \operatorname{Op}\left[\int_{c\left(z_{+}^{l}\right)} e^{i \sigma x_{n}\left(z+\psi^{j l}\right)}\right.$ $\left.g^{l}\left(I-L_{0}(z)\right)^{-1} h \bar{d} z\right]$.

Corollary 3.2. Let $\psi=\psi^{j l}$ and $g=g^{l}$ be the functions in (3.3) and (3.4) respectively. Then there exist a first-order operator $\sum_{p=1}^{n} a_{p} D_{x_{p}}+b\left(b \in \mathcal{B}^{\infty}\right)$ and $a$ second-order operator $\mathcal{L}\left(x, D_{x}\right)$ such that

$$
\begin{aligned}
\left(D_{t}^{2}\right. & \left.-L\left(x, D_{x}\right)\right) \sum_{l=1}^{d} \mathrm{Op}\left[\int_{c_{+}} e^{i \sigma x_{n}\left(z+\psi^{j l}\right)} g^{l}\left(x, \eta^{\prime}, z\right)\left(I-L_{0}\left(x, \eta^{\prime}, z\right)\right)^{-1} h\left(x, \eta^{\prime}\right) \bar{d} z\right] f \\
= & \mathrm{Op}\left[e^{i \sigma x_{n}\left(z_{+}^{j}+\varphi^{j}\right)}\left\{I-L_{0}\left(x, \eta^{\prime}+x_{n} \partial_{x^{\prime}}\left(z_{+}^{j}+\varphi^{j}\right), z_{+}^{j}+\varphi^{j}+x_{n} \partial_{x_{n}}\left(z_{+}^{j}+\varphi^{j}\right)\right)\right\} h \sigma^{2}\right] f \\
& -\mathrm{Op}\left[e^{i \sigma x_{n}\left(z_{+}^{j}+\varphi^{j}\right)}\left(\sum_{p=1}^{n} a_{p} D_{x_{p}}+b\right) h \sigma\right] f-\mathrm{Op}\left[e^{i \sigma x_{n}\left(z_{+}^{j}+\varphi^{j}\right)} \mathcal{L} h\right] f .
\end{aligned}
$$

Proof of Corollary 3.2. Combining Theorem 3.1 and Lemma 2.1, we have

$$
\begin{aligned}
& \left(D_{t}^{2}-L\left(x, D_{x}\right)\right) \sum_{l=1}^{d} \mathrm{Op}\left[\int_{c_{+}} e^{i \sigma x_{n}\left(z+\psi^{j l}\right)} g^{l}\left(x, \eta^{\prime}, z\right)\left(I-L_{0}\left(x, \eta^{\prime}, z\right)\right)^{-1} h\left(x, \eta^{\prime}\right) \bar{d} z\right] f \\
& =\mathrm{Op}\left[e^{i \sigma x_{n}\left(z_{+}^{j}+\varphi^{j}\right)}\left\{I-L_{0}\left(x, \eta^{\prime}+x_{n} \partial_{x^{\prime}}\left(z_{+}^{j}+\varphi^{j}\right), z_{+}^{j}+\varphi^{j}+x_{n} \partial_{x_{n}}\left(z_{+}^{j}+\varphi^{j}\right)\right)\right\}\right. \\
& \quad-\mathrm{Op}\left[e^{i \sigma x_{n}\left(z_{+}^{j}+\varphi^{j}\right)} \sum_{p=1}^{n} a_{p} \sum_{l=1}^{l}\left\{g^{l}\left(z_{+}^{l}\right) R_{0}^{l} h \sigma^{2}\right] f\right. \\
& \left.\quad-\mathrm{Op}\left[e^{l} \tilde{B}_{x_{p}}^{l} h+\left(B_{x_{p}}^{l} g^{l}\right) R_{0}^{l} h\right\} \sigma\right] f \\
& \left.\sum_{l=1} \int_{c_{+}\left(z_{+}^{j}+\varphi^{j}\right)}^{d} L\left\langle g^{l}(z)\left(I-L_{0}(z)\right)^{-1} h\right\rangle \bar{d} z\right] f .
\end{aligned}
$$

Therefore, we obtain Corollary 3.2 since $\sum_{l=1}^{d} g^{l}\left(z_{+}^{l}\right) R_{0}^{l}=I$ (cf. (3.4) and Theorem 2) and $\tilde{B}_{x_{p}}^{l}=D_{x_{p}}+\left(D_{x_{p}} L_{0}\right) R_{1}^{l}$.

Let $\tilde{\eta}^{j}\left(x, \eta^{\prime}\right)$ and $\tilde{z}_{+}^{j}\left(x, \eta^{\prime}\right)$ be $C^{\infty}$ functions satisfying

$$
\left\{\begin{array}{l}
\operatorname{det}\left[I-L_{0}\left(x, \tilde{\eta}^{j}\left(x, \eta^{\prime}\right), \tilde{z}_{+}^{j}\left(x, \eta^{\prime}\right)\right)\right]=0, \\
\tilde{\eta}^{j}\left(x^{\prime}, 0, \eta^{\prime}\right)=\eta^{\prime} \text { and } \tilde{z}_{+}^{j}\left(x^{\prime}, 0, \eta^{\prime}\right)=z_{+}^{j}\left(x^{\prime}, 0, \eta^{\prime}\right) .
\end{array}\right.
$$

When $z_{+}^{j}$ is real (i.e., $j=1, \ldots, k$ ), we take $\tilde{\eta}^{j}$ and $\tilde{z}_{+}^{j}$ trivially so that $\tilde{\eta}^{j}\left(x, \eta^{\prime}\right)=\eta^{\prime}$ and $\tilde{z}_{+}^{j}\left(x, \eta^{\prime}\right)=z_{+}^{j}\left(x, \eta^{\prime}\right)$. The choice of $\tilde{\eta}^{j}$ and $\tilde{z}_{+}^{j}$ is meaningful only in the case of the non-real $z_{+}^{j}$.

We decompose $\mathbb{C}^{n}$ orthogonally into $\operatorname{Ker}\left[I-L_{0}\left(x, \tilde{\eta}^{j}\left(x, \eta^{\prime}\right), \tilde{z}_{+}^{j}\left(x, \eta^{\prime}\right)\right)\right]\left(\equiv K_{j}\right)$ and the orthogonal complement $K_{j}^{\perp}$. Let $P^{j}\left(x, \eta^{\prime}\right)$ be the orthogonal projection to $K_{j}$. Then $\left(I-P^{j}\right)$ becomes the one to $K_{j}^{\perp}$. For the construction of the asymptotic solution, 
we determine $\left\{h_{m}^{j}\right\}_{m=0,1, \ldots}$ by an inductive step for the parts $\left(I-P^{j}\right) h_{0}^{j}(=0), P^{j} h_{0}^{j}$, $\left(I-P^{j}\right) h_{1}^{j}, P^{j} h_{1}^{j}, \cdots$. The following lemma plays a basic role in this step.

LEMma 3.3. Set $\tilde{L}^{j}\left(x, \eta^{\prime}\right)=L_{0}\left(x, \tilde{\eta}^{j}\left(x, \eta^{\prime}\right), \tilde{z}_{+}^{j}\left(x, \eta^{\prime}\right)\right)\left(\left(x, \eta^{\prime}\right) \in U\right)$. Then the following linear operator is non-degenerate.

$$
\left(I-P^{j}\left(x, \eta^{\prime}\right)\right)\left(I-\tilde{L}^{j}\left(x, \eta^{\prime}\right)\right)\left(I-P^{j}\left(x, \eta^{\prime}\right)\right): K_{j}^{\perp} \rightarrow K_{j}^{\perp} .
$$

Proof. Let $n^{\perp}=\operatorname{dim} K_{j}^{\perp}$. Since ${ }^{t} L_{0}=L_{0}$ and $\left(I-\tilde{L}^{j}\right) P^{j}=0$, we have ${ }^{t} P^{j}=P^{j}$ and $P^{j}\left(I-\tilde{L}^{j}\right)={ }^{t}\left(\left(I-\tilde{L}^{j}\right) P^{j}\right)=0$, which yields that $\operatorname{rank}\left[\left(I-P^{j}\right)\left(I-\tilde{L}^{j}\right)(I-\right.$ $\left.\left.P^{j}\right)\right]=\operatorname{rank}\left[\left(I-\tilde{L}^{j}\right)\left(I-P^{j}\right)\right]$. Hence, we have only to shows that $\operatorname{dim}\left[\left(I-\tilde{L}^{j}\right)(I-\right.$ $\left.\left.P^{j}\right) \mathbb{C}^{n}\right] \geq n^{\perp}$. If not, there exist vectors $e_{1}, \ldots, e_{n^{\perp}}$ linearly independent in $K_{j}^{\perp}$ such that $\left(I-\tilde{L}^{j}\right) e_{1}, \ldots,\left(I-\tilde{L}^{j}\right) e_{n^{\perp}}$ are linearly dependent. From the linear dependence, it holds that $\sum_{l=1}^{n^{\perp}} c_{l}\left(I-\tilde{L}^{j}\right) e_{l}=0$ for some numbers $c_{l}$ with a $c_{\tilde{l}} \neq 0$. This means that $\left(I-\tilde{L}^{j}\right)\left(\sum_{l=1}^{n^{\perp}} c_{l} e_{l}\right)=0$. Therefore, we have $\left(\sum_{l=1}^{n^{\perp}} c_{l} e_{l}\right) \in K_{j}$, and namely, $\left(\sum_{l=1}^{n^{\perp}} c_{l} e_{l}\right) \in$ $K_{j} \cap K_{j}^{\perp}=\{0\}$. This is not consistent with the linear independence of $e_{1}, \ldots, e_{n^{\perp}}$. Thus the lemma is proved.

\section{Construction of the surface waves.}

In this section, following the examinations in the previous section, we construct the asymptotic solutions corresponding to the surface wave, i.e., to the residues at the non-real roots $z_{+}^{j}(j=k+1, \ldots, d)$. The construction is performed by determining the series $h_{m}^{j}\left(x, \eta^{\prime}\right)\left(\in \mathcal{B}^{\infty}\left(\overline{\mathbb{R}_{+}^{n}} \times \mathbb{R}^{n-1}\right)\right)$ in the operator $\mathrm{Op}\left[\int_{c_{+}} e^{i \sigma x_{n}\left(z+\psi^{j l}\right)} g^{l}(z)(I-\right.$ $\left.\left.L_{0}(z)\right)^{-1} h_{m}^{j} \sigma^{-m} \bar{d} z\right]$ inductively in $m=0,1,2, \ldots$ for each $j$, where $g^{l}$ is the function defined in (3.4). The procedure is similar to the one for the Poisson operator, and is the same essentially as in Section 4 in Chapter VIII of Taylor [7]. Let the assumptions (A.1)-(A.3) in Introduction be satisfied and (A.4) and (A.5) be done in $U=U_{\left(x^{\prime}, \eta^{\prime}\right)}^{\prime} \times J_{x_{n}}$.

We choose $\varphi^{j}\left(x, \eta^{\prime}\right)$ in (3.3) so that

$$
\left\{\begin{array}{l}
\operatorname{det}\left[I-L_{0}\left(x, \eta^{\prime}+x_{n} \partial_{x^{\prime}}\left(z_{+}^{j}+\varphi^{j}\right), z_{+}^{j}+\varphi^{j}+x_{n} \partial_{x_{n}}\left(z_{+}^{j}+\varphi^{j}\right)\right)\right]=O\left(x_{n}^{\infty}\right), \\
\left.\varphi^{j}\right|_{x_{n}=0}=0
\end{array}\right.
$$

in $U$, where $O\left(x_{n}^{\infty}\right)$ means that $O\left(x_{n}^{\infty}\right) \in \mathcal{B}^{\infty}(\bar{U} \times J)$ and $\left|O\left(x_{n}^{\infty}\right)\right| \leq C_{N} x_{n}^{N}\left(x_{n} \in J\right)$ for any positive integer $N$.

This choice is guaranteed by existence of a function $\tilde{\varphi}$ satisfying

$$
\left\{\begin{array}{l}
\partial_{x_{n}} \tilde{\varphi}-z_{+}^{j}\left(x, \partial_{x^{\prime}} \tilde{\varphi}\right)=O\left(x_{n}^{\infty}\right) \\
\left.\tilde{\varphi}\right|_{x_{n}=0}=x^{\prime} \eta^{\prime}
\end{array}\right.
$$

in $U$. In fact, we can solve this equation (cf. Lemma 3.2 in Soga [6] or Section 4 in Chapter VIII of Taylor [7]), and can get $\varphi^{j}$ by setting $\varphi^{j}=\left(\tilde{\varphi}-x^{\prime} \eta^{\prime}\right) x_{n}^{-1}-z_{+}^{j}$.

Let $\tilde{z}_{+}^{j}\left(x, \eta^{\prime}\right)=z_{+}^{j}\left(x, \eta^{\prime}+x_{n} \partial_{x^{\prime}}\left(z_{+}^{j}\left(x, \eta^{\prime}\right)+\varphi^{j}\left(x, \eta^{\prime}\right)\right)\right)$ and set

$$
\tilde{L}_{0}^{j}\left(x, \eta^{\prime}\right)=L_{0}\left(x, \eta^{\prime}+x_{n} \partial_{x^{\prime}}\left(z_{+}^{j}+\varphi^{j}\right), \tilde{z}_{+}^{j}\right) .
$$


Then, it follows obviously that $\operatorname{det}\left(I-\tilde{L}_{0}^{j}\left(x, \eta^{\prime}\right)\right)=0$ for $\left(x, \eta^{\prime}\right) \in U$.

Lemma 4.1. Let $\tilde{L}_{0}^{j}\left(x, \eta^{\prime}\right)$ be the matrix defined by (4.3). Then we have for $\left(x, \eta^{\prime}\right)$ $\in U$

$$
\tilde{L}_{0}^{j}\left(x, \eta^{\prime}\right)-L_{0}\left(x, \eta^{\prime}+x_{n} \partial_{x^{\prime}}\left(z_{+}^{j}+\varphi^{j}\right), z_{+}^{j}+\varphi^{j}+x_{n} \partial_{x_{n}}\left(z_{+}^{j}+\varphi^{j}\right)\right) \equiv 0 \bmod x_{n}^{\infty} .
$$

Proof. We can write $\operatorname{det}\left[I-L_{0}\left(x, \eta^{\prime}+x_{n} \partial_{x^{\prime}}\left(z_{+}^{j}+\varphi^{j}\right), z\right)\right]=\left(z-\tilde{z}_{+}^{j}\right)^{\alpha^{j}} \gamma(z)$ $\left(\alpha^{j}=\operatorname{mult}\left[\tilde{z}_{+}^{j}\right]\right)$ with $\gamma\left(\tilde{z}_{+}^{j}\right) \neq 0$. This yields that $z-\tilde{z}_{+}^{j}=\left\{\operatorname{det}\left(I-L_{0}\left(x, \eta^{\prime}+x_{n} \partial_{x^{\prime}}\left(z_{+}^{j}+\right.\right.\right.\right.$ $\left.\left.\left.\left.\varphi^{j}\right), z\right)\right) \gamma(z)^{-1}\right\}^{1 / \alpha^{j}}$ near $z=\tilde{z}_{+}^{j}$. Putting $z=z_{+}^{j}+\varphi^{j}+x_{x_{n}} \partial_{x_{n}}\left(z_{+}^{j}+\varphi^{j}\right)$, we have $\left(z_{+}^{j}+\varphi^{j}+x_{n} \partial_{x_{n}}\left(z_{+}^{j}+\varphi^{j}\right)\right)-\tilde{z}_{+}^{j}=O\left(x_{n}^{\infty}\right)$ since $\operatorname{det}\left[I-L_{0}\left(x, \eta^{\prime}+x_{n} \partial_{x^{\prime}}\left(z_{+}^{j}+\varphi^{j}\right), z_{+}^{j}+\right.\right.$ $\left.\left.\varphi^{j}+\partial_{x_{n}}\left(z_{+}^{j}+\varphi^{j}\right) z\right)\right]=O\left(x_{n}^{\infty}\right)\left(\right.$ cf. (4.1)). From the definition of $\tilde{L}_{0}^{j}\left(x, \eta^{\prime}\right)$ it can be written that $\tilde{L}_{0}^{j}\left(x, \eta^{\prime}\right)-L_{0}\left(x, \eta^{\prime}+x_{n} \partial_{x^{\prime}}\left(z_{+}^{j}+\varphi^{j}\right), z_{+}^{j}+\varphi^{j}+x_{n} \partial_{x_{n}}\left(z_{+}^{j}+\varphi^{j}\right)\right)=Q\left(x, \eta^{\prime}\right)\left\{\left(z_{+}^{j}+\varphi^{j}+\right.\right.$ $\left.\left.x_{n} \partial_{x_{n}}\left(z_{+}^{j}+\varphi^{j}\right)\right)-\tilde{z}_{+}^{j}\right\}$ for some $C^{\infty}$ function $Q\left(x, \eta^{\prime}\right)$. Hence, we obtain Lemma 4.1.

The main result in this section is the following theorem.

THEOREM 4.2. Let $\left(\tilde{x}^{\prime}, \tilde{\eta}^{\prime}\right)$ be non-glancing and take a sufficiently small neighborhood $U^{\prime}$ of $\left(\tilde{x}^{\prime}, \tilde{\eta}^{\prime}\right)$. Let $J$ be small enough. We define $\psi^{j l}$ by (3.4) with $\varphi^{j}$ in (4.1) and $g^{l}(l=1, \ldots, d)$ by (3.4). Then, for each $j=k+1, \ldots, d$ there exist matrices $h_{m}^{j}\left(x, \eta^{\prime}\right)$ $\in \mathcal{B}^{\infty}\left(\overline{\mathbb{R}_{+}^{n}} \times \mathbb{R}^{n-1}\right)(m=0,1, \ldots)$ and a neighborhood $\tilde{U}^{\prime}\left(\subset U^{\prime}\right)$ of $\left(\tilde{x}^{\prime}, \tilde{\eta}^{\prime}\right)$ such that the operators

$$
\mathcal{R}_{N}^{j}=\sum_{l=1}^{d} \mathrm{Op}\left[\int_{c_{+}} e^{i \sigma x_{n}\left(z+\psi^{j l}\right)} g^{l}\left(I-L_{0}(z)\right)^{-1} \sum_{m=0}^{N} h_{m}^{j} \sigma^{-m} \bar{d} z\right]
$$

satisfy the following (i)-(iii) for any non-negative integer $N$.

(i) $\left(D_{t}^{2}-L\left(x, D_{x}\right)\right) \mathcal{R}_{N}^{j}$ is a bounded operator from $H^{s}$ to $\mathcal{H}^{s-2, s+N-1}(s=2,3, \ldots)$.

(ii) Each column of $\left.h_{m}^{j}\right|_{x_{n}=0}(m=0,1, \ldots)$ can be chosen arbitrarily in the space $\left.K_{j}\right|_{x_{n}=0}$ for $\left(x^{\prime}, \eta^{\prime}\right) \in \tilde{U}^{\prime}$, where $K_{j}=\operatorname{Ker}\left[I-\tilde{L}_{0}^{j}\left(x, \eta^{\prime}\right)\right]$.

(iii) For any $f \in H^{0}$ and $N \geq 0,\left(\mathcal{R}_{N}^{j} f\right)(t, x)$ is $C^{\infty}$-smooth if $x_{n}>0$.

Proof. Setting $h_{-2}^{j}=h_{-1}^{j}=0$, by Corollary 3.2 and Lemma 4.1 we can write

$$
\begin{aligned}
& \left(D_{t}^{2}-L\left(x, D_{x}\right)\right) \mathcal{R}_{N}^{j} f \\
& =\mathrm{Op}\left[e ^ { i \sigma x _ { n } ( z _ { + } ^ { j } + \varphi ^ { j } ) } \left\{\sum_{m=0}^{N}\left(P^{j}\left(I-\tilde{L}_{0}^{j}\right)\left(I-P^{j}\right) h_{m}^{j}+\left(I-\tilde{L}_{0}^{j}\right) P^{j} h_{m}^{j}+O\left(x_{n}^{\infty}\right) h_{m}^{j}\right) \sigma^{-m+2}\right.\right. \\
& \quad+\sum_{m=-1}^{N-1}\left(\left(I-P^{j}\right)\left(I-\tilde{L}_{0}^{j}\right)\left(I-P^{j}\right) h_{m+1}^{j}\right. \\
& \left.\quad-\left(I-P^{j}\right)\left(\sum_{p=1}^{n} a_{p} D_{x_{p}}+b\right) h_{m}^{j}-\left(I-P^{j}\right) \mathcal{L} h_{m-1}^{j}\right) \sigma^{-m+1}
\end{aligned}
$$




$$
\begin{aligned}
& -\sum_{m=0}^{N}\left(P^{j}\left(\sum_{p=1}^{n} a_{p} D_{x_{p}}+b\right) P^{j} h_{m}^{j}\right. \\
& \left.\quad+P^{j}\left(\sum_{p=1}^{n} a_{p} D_{x_{p}}+b\right)\left(I-P^{j}\right) h_{m}^{j}+P^{j} \mathcal{L} h_{m-1}^{j}\right) \sigma^{-m+1} \\
& \left.\left.-\left(\left(I-P^{j}\right)\left(\sum_{p=1}^{n} a_{p} D_{x_{p}}+b\right) h_{N}^{j}+\left(I-P^{j}\right) \mathcal{L} h_{N-1}^{j}\right) \sigma^{-N+1}-\mathcal{L} h_{N}^{j} \sigma^{N}\right\}\right] f .
\end{aligned}
$$

In view of the equality $P^{j}\left(I-\tilde{L}_{0}^{j}\right)=\left(I-\tilde{L}_{0}^{j}\right) P^{j}=0$ and (ii) of Lemma 2.4 , we have only to choose $\left\{h_{m}^{j}\right\}$ so that the above summations of the orders $-m$ and $-m+1$ in $\sigma$ are eliminated in the right hand of the equality (4.5). This is reduced to solving the linear algebraic equation

$$
\left(I-P^{j}\left(x, \eta^{\prime}\right)\right)\left(I-\tilde{L}_{0}^{j}\left(x, \eta^{\prime}\right)\right)\left(I-P^{j}\left(x, \eta^{\prime}\right)\right) h=\left(I-P^{j}\left(x, \eta^{\prime}\right)\right) \tilde{h}
$$

for any given $\tilde{h} \in \mathbb{C}^{n}$ and the differential equation

$$
\left\{\begin{array}{l}
P^{j}\left(\sum_{p=1}^{n} a_{p} D_{x_{p}}+b\right) P^{j} h=P^{j} \tilde{h}, \\
\left.P^{j} h\right|_{x_{n}=0}=\left.P^{j} \tilde{f}\right|_{x_{n}=0} \text { for }\left(x^{\prime}, \eta^{\prime}\right) \in U^{\prime}
\end{array}\right.
$$

for any given $\tilde{h} \in \mathcal{B}^{\infty}$ and with any boundary value $\left.P^{j} \tilde{f}\right|_{x_{n}=0}$.

Lemma 3.3 guarantees the solvability of (4.6). Let us consider (4.7). We take orthogonal bases $\left\{e_{l}\left(x, \eta^{\prime}\right)\right\}_{l=1, \ldots, n^{j}}$ in $K_{j}$. Here we can assume that $e_{l}\left(x, \eta^{\prime}\right) \in \mathcal{B}^{\infty}\left(\overline{\mathbb{R}_{+}^{n}} \times\right.$ $\left.\mathbb{R}^{n-1}\right)$. Set $e=\left(e_{1}, \ldots, e_{n^{j}}\right)$. Expressing vectors in $K_{j}$ by linear combination of the bases $\left\{e_{l}\right\}_{l=1, \ldots, n^{j}}$ (i.e., using the local coordinates: $\left.\mathbb{C}^{n^{j}} \ni w={ }^{t}\left(w_{1}, \ldots, w_{n^{j}}\right) \mapsto e w\left(\in K_{j}\right)\right)$, we transform the equation (4.7) into the equation

$$
\left\{\begin{array}{l}
\sum_{p=1}^{n}{ }^{t} \bar{e} a_{p} e D_{x_{p}} w+b^{\prime} w={ }^{t} \bar{e} \tilde{h} \\
\left.w\right|_{x_{n}=0}={ }^{t} \bar{e} \tilde{f}
\end{array}\right.
$$

where $b^{\prime}=\sum_{p=1}^{n}{ }^{t} \bar{e} a_{p} D_{x_{p}} e+b$. Since $\left.\operatorname{Im}\left(a_{n} v, v\right)\right|_{x_{n}=0}=\left.2\left(\operatorname{Im} z_{+}^{j}\right)\left(a_{n n} v, v\right)\right|_{x_{n}=0}$ (cf. the definition of $a_{n}$ in Theorem 3.1) and $\left.\inf _{\left(x^{\prime}, \eta^{\prime}\right) \in U^{\prime}} \operatorname{Im} z_{+}^{j}\right|_{x_{n}=0}>0$, the coefficient ${ }^{t} \bar{e} a_{n} e$ in (4.8) is a non-degenerate matrix. Therefore, we can solve (4.8) modulo $x_{n}^{\infty}$ in the same way as Taylor [6] did (cf. Section 4 in Chapter VIII). Thus we can determine $\left(I-P^{j}\right) h_{0}^{j}$ $(=0), P^{j} h_{0}^{j},\left(I-P^{j}\right) h_{1}^{j}, P^{j} h_{1}^{j}, \ldots$ inductively. Hence, Theorem 4.2 is obtained.

\section{Construction of the body waves and proof of Theorem 1 .}

In this section we construct the asymptotic solutions $\sum_{l=1}^{d} \mathrm{Op}\left[\int_{c_{+}} e^{i \sigma x_{n}\left(z+\psi^{j l}\right)} g^{l}(I-\right.$ $\left.\left.L_{0}(z)\right)^{-1} \sum_{m=0}^{\infty} h_{m}^{j} \sigma^{-m} \bar{d} z\right]$ corresponding to the body waves, i.e., to the residues at the real roots $z_{+}^{j}(j=1, \ldots, k)$. Furthermore, we prove Theorem 1 in Introduction 
(Section 1). The procedure of the construction is similar to the case of the surface waves (in Section 4). However, we need to solve the equations for $h_{m}^{j}$ exactly, not 'modulo $x_{n}^{\infty}$ ', and also the equation for $P^{j} h_{m}^{j}$ is of the different type.

Fix $j(=1, \ldots, k)$, and for $l=1, \ldots, d$ set

$$
\psi^{j l}\left(x, \eta^{\prime}\right)=z_{+}^{j}\left(x, \eta^{\prime}\right)+\varphi^{j}\left(x, \eta^{\prime}\right)-z_{+}^{l}\left(x, \eta^{\prime}\right) .
$$

Here, $\varphi^{j}$ is chosen so that it satisfies exactly the equations $\operatorname{det}\left\{I-L_{0}\left(x, \eta^{\prime}+x_{n} \partial_{x^{\prime}}\left(z_{+}^{j}+\right.\right.\right.$ $\left.\left.\left.\varphi^{j}\right), z_{+}^{j}+\varphi^{j}+x_{n} \partial_{x_{n}}\left(z_{+}^{j}+\varphi^{j}\right)\right)\right\}=0$ and $\left.\varphi^{j}\right|_{x_{n}=0}=0$. Existence of this $\varphi^{j}$ is reduced to solvability of the equation

$$
\left\{\begin{array}{l}
\partial_{x_{n}} \psi-z_{+}^{j}\left(x, \partial_{x^{\prime}} \psi\right)=0, \quad x_{n} \in J \\
\left.\psi\right|_{x_{n}=0}=x^{\prime} \eta^{\prime}
\end{array}\right.
$$

Here, let us note that $z_{+}^{j}\left(x, \eta^{\prime}\right)$ can be assumed to be real-valued and to belong to $\mathcal{B}^{\infty}\left(\overline{\mathbb{R}_{+}^{n}} \times \mathbb{R}^{n-1}\right)$ since mult $\left[z_{+}^{j}\left(x, \eta^{\prime}\right)\right]\left(=\operatorname{mult}\left[\lambda_{j}\left(x, \eta^{\prime}, z_{+}^{j}\left(x, \eta^{\prime}\right)\right]\right)\right.$ is constant in a small neighborhood $U=U_{\left(x^{\prime}, \eta^{\prime}\right)}^{\prime} \times J_{x_{n}}$. By the Hamiton-Jacobi method we can solve the equation (5.1) exactly (not modulo $x_{n}^{\infty}$ ) (e.g., cf. Theorem 4.1 in Chapter 10 of Kumano-go $[3])$ and get the required $\varphi^{j}$ by setting $\varphi^{j}=\left(\psi-x^{\prime} \eta^{\prime}\right) x_{n}^{-1}-z_{+}^{j}$.

Defining $\psi^{j l}$ and $\varphi^{j}$ in the way stated above and $g^{l}$ by (3.4), we obtain the following theorem.

TheOrem 5.1. Let $\left(\tilde{x}^{\prime}, \tilde{\eta}^{\prime}\right)$ be non-glancing and take a sufficiently small neighborhood $U^{\prime}$ of $\left(\tilde{x}^{\prime}, \tilde{\eta}^{\prime}\right)$. Let $J_{x_{n}}=[0, r]$ be small enough. Then, for each $j=1, \ldots, k$ there exist matrices $h_{m}^{j}\left(x, \eta^{\prime}\right) \in \mathcal{B}^{\infty}\left(\overline{\mathbb{R}_{+}^{n}} \times \mathbb{R}^{n-1}\right)(m=0,1, \ldots)$ and a neighborhood $\tilde{U}^{\prime}\left(\subset U^{\prime}\right)$ of $\left(\tilde{x}^{\prime}, \tilde{\eta}^{\prime}\right)$ such that the operator

$$
\mathcal{R}_{N}^{j}=\sum_{l=1}^{d} \mathrm{Op}\left[\int_{c_{+}} e^{i \sigma x_{n}\left(z+\psi^{j l}\right)} g^{l}\left(I-L_{0}(z)\right)^{-1} \sum_{m=0}^{N} h_{m}^{j} \sigma^{-m} d z\right]
$$

satisfies the following (i) and (ii) for any non-negative integer $N$.

(i) $\left(D_{t}^{2}-L\left(x, D_{x}\right)\right) \mathcal{R}_{N}^{j}$ is a bounded operator from $H^{s}$ to $\mathcal{H}^{s-2, s+N-1}(s=2,3, \ldots)$.

(ii) Each column of $\left.h_{m}^{j}\right|_{x_{n}=0}(m=0,1, \ldots)$ can be chosen arbitrarily in the space $\left.K_{j}\right|_{x_{n}=0}$ for $\left(x^{\prime}, \eta^{\prime}\right) \in \tilde{U}^{\prime}$, where $K_{j}=\operatorname{Ker}\left[I-L_{0}\left(x, \eta^{\prime}+x_{n} \partial_{x^{\prime}}\left(z_{+}^{j}+\varphi^{j}\right), z_{+}^{j}+\varphi^{j}+\right.\right.$ $\left.\left.x_{n} \partial_{x_{n}}\left(z_{+}^{j}+\varphi^{j}\right)\right)\right]$.

Proof. We reform $\left(D_{t}^{2}-L\right) \mathcal{R}_{N}^{j} f$ in the same way as (4.5) in Section 4 . In the case of Theorem 5.1, we define $\tilde{L}^{j}\left(x, \eta^{\prime}\right)$ in Lemma 3.3 so that $\tilde{L}^{j}\left(x, \eta^{\prime}\right)=L_{0}\left(x, \eta^{\prime}+\right.$ $x_{n} \partial_{x^{\prime}}\left(z_{+}^{j}+\varphi^{j}\right), z_{+}^{j}+\varphi^{j}+x_{n} \partial_{x_{n}}\left(z_{+}^{j}+\varphi^{j}\right)$ ) (cf. (4.3)). This is because the equation (5.1) is solved exactly (not ' $\bmod x_{n}^{\infty}$ ) (cf. (4.2)). Therefore, the term $O\left(x_{n}^{\infty}\right)$ in (4.5) is dropped in this case.

We determine $\left(I-P^{j}\right) h_{0}^{j}(=0), P^{j} h_{0}^{j},\left(I-P^{j}\right) h_{1}^{j}, P^{j} h_{1}^{j}, \ldots$ inductively by solving the equations corresponding to (4.6) and (4.7). The idea of this procedure is the same 
essentially as in Lax [4]. The treatment for $\left(I-P^{j}\right) h_{m}^{j}$ is the same that for Theorem 4.1. However, $P^{j} h_{m}^{j}$ is determined differently.

Taking orthonormal bases $e_{1}, \ldots, e_{n^{j}}$ in $K_{j}=\operatorname{Ker}\left(I-\tilde{L}^{j}\right)$ in the same way as for the equation (4.7), we transform the equations for $P^{j} h_{m}^{j}$ into the following form.

$$
\left\{\begin{array}{l}
\sum_{p=1}^{n}{ }^{t} \bar{e} a_{p} e D_{x_{p}} w+b^{\prime} w={ }^{t} \bar{e} \tilde{h} \\
\left.w\right|_{x_{n}=0}={ }^{t} \bar{e} \tilde{f}
\end{array}\right.
$$

where $e=\left(e_{1}, \ldots, e_{n^{j}}\right)$. This equation is of the real symmetric type. If the coefficient ${ }^{t} \bar{e} a_{n} e$ is non-degenerate, we get the solution of (5.2) for any $\tilde{f}$ and $\tilde{h}$ by the method in Friedrichs [1]. This non-degeneracy is guaranteed by Lemma 5.2 below. Therefore, we obtain Theorem 5.1 by the same procedure as for Theorem 4.1.

The coefficient $a_{n}$ in (5.2) is of the form $\left.\sum_{p=1}^{n}\left(a_{p n}+a_{n p}\right) \eta_{p}\right|_{x_{n}=0}$ at $x_{n}=0$ (see the definition of $a_{n}$ in Theorem 3.1). The following lemma means that $\left.{ }^{t} \bar{e} a_{n} e\right|_{x_{n}=0}$ is non-degenerate.

Lemma 5.2. Let $\left(x^{\prime}, \eta^{\prime}\right)$ be in $U^{\prime}$. Then, for $j=1, \ldots, k$ the following linear operator is non-degenerate.

$$
\left.\sum_{p=1}^{n}\left(a_{p n}+a_{n p}\right) \eta_{p}\right|_{x_{n}=0}:\left.\left.K_{j}\right|_{x_{n}=0} \rightarrow K_{j}\right|_{x_{n}=0} \quad\left(\eta_{n}=z_{+}^{j}\right) .
$$

Proof. Let $z(\in \mathbb{R})$ move near $z_{+}^{j}$ and let $P(z)$ be the orthogonal projection to the eigen-space of the eigen-value $\lambda_{j}\left(x^{\prime}, 0, z\right)$. Then it follows that

$$
P(z)\left(I-L_{0}(z)\right) P(z)=\left(1-\lambda_{j}(z)\right) P(z) .
$$

Differentiating this equality in $z$, we have

$$
\left(\partial_{z} P\right)\left(I-L_{0}\right) P+P \sum_{p=1}^{n}\left(a_{p n}+a_{n p}\right) \eta_{p} P+P\left(I-L_{0}\right) \partial_{z} P=-\left(\partial_{z} \lambda_{j}\right) P+\left(1-\lambda_{j}\right) \partial_{z} P
$$

where $\eta_{n}=z$. Noting that $P\left(z_{+}^{j}\right)\left(I-L_{0}\left(z_{+}^{j}\right)\right)=\left(I-L_{0}\left(z_{+}^{j}\right)\right) P\left(z_{+}^{j}\right)=(1-$ $\left.\lambda_{j}\left(z_{+}^{j}\right)\right) P\left(z_{+}^{j}\right)=0$, we obtain

$$
P\left(z_{+}^{j}\right) \sum_{p=1}^{n}\left(a_{p n}+a_{n p}\right) \eta_{p} P\left(z_{+}^{j}\right)=-\left(\partial_{z} \lambda_{j}\right)\left(z_{+}^{j}\right) P\left(z_{+}^{j}\right) .
$$

Therefore, Lemma 5.2 is obtained since $\partial_{z} \lambda_{j}\left(z_{+}^{j}\right) \neq 0$.

Let us prove Theorem 1 in Introduction (Section 1) at the end of this section.

Proof of Theorem 1. Let $\mathcal{R}_{N}^{j}$ be the operators Theorems 4.2 and 5.1. In these theorems we have shown that $\left(D_{t}^{2}-L\right) \mathcal{R}_{N}^{j}$ is a bounded operator from $H^{s}$ to $\mathcal{H}^{s-2, s+N-1}$, 
and that each column of $\left.h_{m}^{j}\right|_{x_{n}=0}$ in $\mathcal{R}_{N}^{j}$ can be chosen arbitrarily in $\left.K_{j}\right|_{x_{n}=0}$. Let us note that $\sum_{j=1}^{d} \mathcal{R}_{N}^{j} f$ is of the form $\sum_{j=1}^{d} \sum_{l=1}^{d} \mathrm{Op}\left[\int_{c_{+}} e^{i \sigma x_{n}\left(z+\psi^{j l}\left(x, \eta^{\prime}\right)\right)} g^{l}\left(x, \eta^{\prime}, z\right)(I-\right.$ $\left.\left.L_{0}\left(x, \eta^{\prime}, z\right)\right)^{-1} \sum_{m=0}^{N} h_{m}^{j}\left(x, \eta^{\prime}\right) \sigma^{-m} \bar{d} z\right] f$.

Let $G^{j}$ be the matrix in (2.9) (note that ${ }^{t} G^{j}$ is equal to $M^{j}$ in Theorem 2). Since $\left.R_{0}^{j} \mathbb{C}^{n}\right|_{x_{n}=0}=\left.K_{j}\right|_{x_{n}=0}\left(R_{0}^{j}=\operatorname{Res}_{z=z_{+}^{j}}(I-L(z))^{-1}\right)$, each column of the matrix $\left.R_{0}^{j} G^{j}\right|_{x_{n}=0}$ belongs to $\left.K_{j}\right|_{x_{n}=0}$. Noting this, we choose $\left.h_{0}^{j}\right|_{x_{n}=0}=\left.R_{0}^{j} G^{j}\right|_{x_{n}=0}$ and $\left.h_{m}^{j}\right|_{x_{n}=0}=0$ for $m=1,2, \ldots$. Then, if $\mathrm{WF}[f]$ is contained in $\left\{\left(t, x^{\prime}, \sigma, \xi^{\prime}\right)\right.$ $\left.\chi\left(x^{\prime}, 0, \sigma, \xi^{\prime} / \sigma\right)=1, \sigma>0\right\}$, by $(2.9)$ we have $\left.\sum_{j=1}^{d} \mathcal{R}_{N}^{j} f\right|_{x_{n}=0}=f \bmod C^{\infty}$.

Theorem 4.2 shows that $\operatorname{sing} \operatorname{supp}\left[\mathcal{R}_{N}^{j} f\right](j=k+1, \ldots, d)$ is only in the boundary $\mathbb{R}_{t}^{1} \times \mathbb{R}_{x^{\prime}}^{n-1}$ and is contained in sing supp $[f]$. Furthermore, Theorem 6.2 in the next section implies that if sing supp $[f] \subset\left\{\left(t, x^{\prime}\right) \mid t \geq t_{0}\right\}, \operatorname{sing} \operatorname{supp}\left[\mathcal{R}_{N}^{j} f\right](j=1, \ldots, k)$ is contained in $\left\{\left(t, x^{\prime}, x_{n}\right) \mid t \geq t_{0}+\varepsilon x_{n}\right\}$ for some positive constant $\varepsilon$. Hence, $\mathcal{R}_{N}^{j} f(j=1, \ldots, k)$ is outgoing.

Let us prove the last statement in Theorem 1; namely, we can reform the principal part of $\mathcal{R}_{N}^{j}$ in the way stated in this theorem. As is stated in the proofs of Theorems 4.2 and 5.1 , the matrices $h_{m}^{j}(m=0,1, \ldots)$ are determined through the inductive processes for the terms $\left(I-P^{j}\right) h_{m}^{j}$ and $P^{j} h_{m}^{j}(m=0,1, \ldots)$ with $\left(I-P^{j}\right) h_{0}^{j}=0$, where $P^{j}$ is the orthogonal projection to $K_{j}$. Hence, the principal part $\left(=\mathcal{R}_{0}^{j}\right)$ is of the form

$$
\mathcal{R}_{0}^{j}=\mathrm{Op}\left[\sum_{l=1}^{d} e^{i \sigma x_{n}\left(z_{+}^{l}+\psi^{l j}\right)} g^{l}\left(z_{+}^{l}\right) R_{0}^{l} h_{0}^{j}\right]=\mathrm{Op}\left[e^{i \sigma x_{n}\left(z_{+}^{j}+\varphi^{j}\right)} P^{j} h_{0}^{j}\right]
$$

(cf. the choices of $\psi^{l j}$ and $g^{l}(z)$, i.e., (3.3) and (3.4)). Noting that $K_{j}=R_{0}^{j} \mathbb{C}^{n}$, for the orthonormal bases $\left\{e_{l}^{j}\right\}_{l=1, \ldots, n^{j}}$ in $K_{j}$ we have vectors $\tilde{e}_{l}^{j}$ such that $e_{l}^{j}=R_{0}^{j} \tilde{e}_{l}^{j}$. On the other hand $P^{j}$ is expressed of the form $P^{j}=e^{j t} \bar{e}^{j}=R_{0}^{j} \tilde{e}^{j t} \bar{e}^{j}$, where $e^{j}=\left(e_{1}^{j}, \ldots, e_{n^{j}}^{j}\right)$ and $\tilde{e}^{j}=\left(\tilde{e}_{1}^{j}, \ldots, \tilde{e}_{n^{j}}^{j}\right)$. Set

$$
\tilde{g}_{0}^{j}\left(x, \eta^{\prime}, z\right)=\rho^{j}\left(x, \eta^{\prime}, z\right) I, \quad \tilde{h}_{0}^{j}\left(x, \eta^{\prime}\right)=\tilde{e}^{j}\left(x, \eta^{\prime}\right)^{t} \overline{e^{0}}\left(x, \eta^{\prime}\right) h_{0}^{j},
$$

where $\rho^{j}$ is the function (3.2). Then we have

$$
\begin{aligned}
& \mathrm{Op}\left[\int_{c_{+}} e^{i \sigma x_{n}\left(z+\varphi^{j}\right)} \tilde{g}_{0}^{j}(z)\left(I-L_{0}(z)\right)^{-1} \tilde{h}_{0}^{j} d z\right] \\
& \quad=\mathrm{Op}\left[e^{i \sigma x_{n}\left(z_{+}^{j}+\varphi^{j}\right)} R_{0}^{j} \tilde{h}_{0}^{j}\right]=\mathrm{Op}\left[e^{i \sigma x_{n}\left(z_{+}^{j}+\varphi^{j}\right)} P^{j} h_{0}^{j}\right]
\end{aligned}
$$

which is equal to $\mathcal{R}_{0}^{j}$. Thus the required form is obtained.

\section{Singularities of the solutions.}

In this section we examine singularities of the outgoing solutions to the equation (1.1). Namely we estimate the wave front set (denoted by WF) of the solution $u(x, t)$ by the wave front set of the (non-glancing) boundary value $f\left(x^{\prime}, t\right)$ (for the definition of WF, see Section 3 in Chapter 10 of $[\mathbf{3}])$. Let us note that, in general, sing $\operatorname{supp}[f(X)]=\{X \mid(X, \Xi) \in \mathrm{WF}[f]$ for some $\Xi\}$. A series of the forms 
$\left\{\sum_{j=1}^{d} \mathcal{R}_{N}^{j} f\right\}_{N=0,1, \ldots}$ constructed in the previous sections yields the asymptotic expansion of the outgoing (genuine) solution $u$ with the boundary value $f$ when $x_{n} \in J$. Therefore, $\mathrm{WF}[u]$ in $J$ is known by $\bigcup_{N=1}^{\infty} \sum_{j=1}^{d} \mathrm{WF}\left[\mathcal{R}_{N}^{j} f\right]$. As is described later, we can construct operators $\mathcal{R}_{\infty}^{j}$ with the asymptotic expansion $\sum_{m=0}^{N} \mathcal{R}_{m}^{j}(N=0,1, \ldots)$ such that $\sum_{j=1}^{d} \mathcal{R}_{\infty}^{j} f$ is equal to $u$ modulo $C^{\infty}$ functions (cf. (6.3)). We note also that WF $[u]$ in $\left\{-\infty<t<t_{0}+\delta\right\} \times \mathbb{R}_{x^{\prime}}^{n-1} \times\left\{0 \leq x_{n}<\infty\right\}$ (for some $\delta>0$ ) is estimated by $\mathcal{R}_{\infty}^{j}$ (i.e., $\left.\mathcal{R}_{N}^{j}\right)$.

$\mathrm{WF}\left[\sum_{j=1}^{d} \mathcal{R}_{N}^{j} f\right]$ is determined only by $\mathcal{R}_{N}^{j} f$ for $j=1,2, \ldots, k$ since $\mathcal{R}_{N}^{j} f$ for $j=$ $k+1, \ldots, d$ are $C^{\infty}$ smooth when $x_{n}>0$ (cf. Theorem 4.1). Taking the residues of the Cauchy integral, we can rewrite $\mathcal{R}_{N}^{j} f$ for $j=1, \ldots, k$ to the forms of the Fourier integral operators obtained in Soga [6]. On the other hand, the wave front sets for the Fourier integral operators have been examined as general theories, e.g., see Section 5 of Kumano-go [3]. Hence, combining these general theories and the expressions of the solutions in Soga [6], we can obtain concrete estimation of the wave front sets of the solutions like Theorem 6.2 and Corollary 6.3 stated below. However, we remark that our asymptotic solutions give an integral form unifying all the modes (i.e. the body waves and the surface waves) and have accuracy equivalent to the forms by Soga $[\mathbf{6}]$ and others.

At first we mention the general theory in the book of Kumano-go [3] concerning the wave front set. Let $\phi(X, \Xi)$ be a real-valued $C^{\infty}$ function on $\mathbb{R}_{X}^{\tilde{n}} \times \mathbb{R}_{\Xi}^{\tilde{n}}$ homogeneous of order 1 in $\Xi(|\Xi| \geq 1)$ and satisfying

$$
\left|\partial_{\Xi}^{\alpha} \partial_{X}^{\beta}(\phi(X, \Xi)-X \Xi)\right| \leq \tau(1+|\Xi|)^{1-|\alpha|}
$$

for any $\alpha$ and $\beta$ with $|\alpha+\beta| \leq 2$ and some constant $\tau$ with $0 \leq \tau<1$. Then the mapping: $X \mapsto \partial_{\Xi} \phi(X, \Xi)$ is a diffeomorphism from $\mathbb{R}^{\tilde{n}}$ to $\mathbb{R}^{\tilde{n}}$. Therefore, for any $\tilde{X} \in \mathbb{R}^{\tilde{n}}$ and $\tilde{\Xi} \in \mathbb{R}^{\tilde{n}}$ there exists an $X \in \mathbb{R}^{\tilde{n}}$ uniquely such that $\tilde{X}=\partial_{\tilde{\Xi}} \phi(X, \tilde{\Xi})$. We employ the mapping $T:(\tilde{X}, \tilde{\Xi}) \mapsto\left(X, \partial_{X} \phi(X, \tilde{\Xi})\right)$.

We consider the Fourier integral operator $P_{\phi}$

$$
P_{\phi} f(X)=\int e^{i \phi(X, \Xi)} p(X, \Xi) \hat{f}(\Xi) d \Xi .
$$

Then we have a result concerning $\mathrm{WF}\left[P_{\phi} f\right]$ described in Kumano-go [3] (cf. Theorem 3.14 in Chapter 10):

Proposition 6.1. For open sets $V$ in $\mathbb{R}_{X}^{\tilde{n}} \times \mathbb{R}_{\Xi}^{\tilde{n}}$ we denote the smallest conic neighborhoods of $V$ (in $\Xi)$ by $V^{\text {con }}$. Then we have

$$
\mathrm{WF}\left[P_{\phi} f\right] \subset\{(X, \Xi)=T(\tilde{X}, \tilde{\Xi}) \mid(\tilde{X}, \tilde{\Xi}) \in \mathrm{WF}[f]\}^{c o n} .
$$

Furthermore, it is known also that when $\phi$ has a parameter, $\mathrm{WF}\left[P_{\phi} f\right]$ consists of the curves associated with that parameter (e.g., the bicharacteristic curves connected with the initial values or boundary values, etc.).

Using Proposition 6.1, we can estimate the wave front set of $\mathcal{R}_{N}^{j} f$ defined in the previous sections: 
TheOREM 6.2. Let $\mathcal{R}_{N}^{j}(j=1, \ldots, d)$ be the operators constructed in Theorems 4.2 and 5.1, and let $\mathrm{WF}[f]\left(f \in H^{2}\right)$ be contained in $\left\{\left(t, x^{\prime}, \sigma, \xi^{\prime}\right) \mid \chi\left(x^{\prime}, 0, \sigma, \xi^{\prime} / \sigma\right)=1\right\}$ (where $\chi$ is the cutoff function in $\mathcal{R}_{N}^{j}$ ). Then the wave front set of $\left[\mathcal{R}_{N}^{j} f\left(x_{n}\right)\right]\left(t, x^{\prime}\right)$ is estimated at each $x_{n} \in J$ as follows:

(i) For $j=1, \ldots, k, \mathrm{WF}\left[\mathcal{R}_{N}^{j} f\left(x_{n}\right)\right]$ is contained in

$$
\begin{aligned}
\left\{\left(t, x^{\prime}, \tilde{\sigma}, \xi^{\prime}\right) \mid\right. & t=\tilde{t}-\left(\partial_{\xi_{n}} \lambda_{j}\left(\tilde{x}^{\prime}, 0, \tilde{\eta}^{\prime}, z_{+}^{j}\right)\right)^{-1} x_{n}+O\left(x_{n}^{2}\right), \\
& x^{\prime}=\tilde{x}^{\prime}+\left(\partial_{\xi_{n}} \lambda_{j}\left(\tilde{x}^{\prime}, 0, \tilde{\eta}^{\prime}, z_{+}^{j}\right)\right)^{-1} x_{n} \partial_{\xi^{\prime}} \lambda_{j}\left(\tilde{x}^{\prime}, 0, \tilde{\eta}^{\prime}, z_{+}^{j}\right)+O\left(x_{n}^{2}\right), \\
& \xi^{\prime}=\tilde{\xi}^{\prime}-\tilde{\sigma}\left(\partial_{\xi_{n}} \lambda_{j}\left(\tilde{x}^{\prime}, 0, \tilde{\eta}^{\prime}, z_{+}^{j}\right)\right)^{-1} x_{n} \partial_{x^{\prime}} \lambda_{j}\left(\tilde{x}^{\prime}, 0, \tilde{\eta}^{\prime}, z_{+}^{j}\right)+\tilde{\sigma} O\left(x_{n}^{2}\right), \\
& \left.\left(\tilde{t}, \tilde{x}^{\prime}, \tilde{\sigma}, \tilde{\xi}^{\prime}\right) \in \mathrm{WF}[f] \quad\left(\tilde{\eta}^{\prime}=\tilde{\xi}^{\prime} / \tilde{\sigma}\right)\right\}^{c o n},
\end{aligned}
$$

where $z_{+}^{j}=z_{+}^{j}\left(\tilde{x}^{\prime}, 0, \tilde{\eta}^{\prime}\right)$ and $O\left(x_{n}^{2}\right)$ denotes some $C^{\infty}$ function with the $C^{l}$-norms $\leq C_{l} x_{n}^{2}\left(x_{n} \in J\right)$.

(ii) For $j=k+1, \ldots, d, \operatorname{WF}\left[\mathcal{R}_{N}^{j} f\left(x_{n}\right)\right]$ is empty if $x_{n}>0$, and is equal to $\mathrm{WF}[f]$ if $x_{n}=0$.

Let $\left\{h_{m}^{j}\right\}_{m=0,1, \ldots}$ be the matrices in $\mathcal{R}_{N}^{j}$, and consider the sum

$$
\sum_{m=0}^{\infty} h_{m}^{j}\left(x, \eta^{\prime}\right) \chi_{m}(\sigma) \sigma^{-m}
$$

where $\chi_{m}(\sigma)=\tilde{\chi}\left(|\sigma|-\sigma_{m}\right), \tilde{\chi}(\tilde{\sigma})$ is a $C^{\infty}$ function equal to 0 for $\tilde{\sigma}<1 / 2$ and to 1 for $\tilde{\sigma}>$ 1 and $\left\{\sigma_{m}\right\}_{m=0,1, \ldots}$ is an appropriate sequence satisfying $\lim _{m \rightarrow \infty} \sigma_{m}=+\infty$. Then the sum (6.2) converges and belongs to the symbol class $S_{1,0}^{0}$ with $\eta^{\prime}=\xi^{\prime} / \sigma$ if $\left\{\sigma_{m}\right\}_{m=0,1, \ldots}$ increases sufficiently. Furthermore, it has the asymptotic expansion $\sum_{m=0}^{\infty} h_{m}^{j}\left(x, \eta^{\prime}\right) \sigma^{-m}$, i.e., for any $N=1,2, \ldots$

$$
\left|\partial_{x}^{\alpha} \partial_{\left(\sigma, \eta^{\prime}\right)}^{\beta}\left(\sum_{m=0}^{\infty} h_{m}^{j} \chi_{m} \sigma^{-m}-\sum_{m=0}^{N-1} h_{m}^{j} \sigma^{-m}\right)\right| \leq C_{\alpha \beta}|\sigma|^{-N} \quad(|\sigma|>1) .
$$

We define the operator $\mathcal{R}_{\infty}^{j}$ by

$$
\mathcal{R}_{\infty}^{j}=\sum_{l=1}^{d} \mathrm{Op}\left[\int_{c_{+}} e^{i \sigma x_{n}\left(z+\psi^{j l}\right)} g^{l}\left(I-L_{0}(z)\right)^{-1} \sum_{m=0}^{\infty} h_{m}^{j} \chi_{m} \sigma^{-m} \overline{d z}\right] .
$$

Then $\mathrm{WF}\left[\mathcal{R}_{\infty}^{j} f\right]$ coincides with $\bigcup_{N=1}^{\infty} \mathrm{WF}\left[\mathcal{R}_{N}^{j} f\right]$ for each $x_{n} \in J$, and $\mathcal{R}_{\infty}^{j} f$ satisfies $\left(D_{t}^{2}-L\right) \mathcal{R}_{\infty}^{j} f \in C^{0}\left(J_{x_{n}} ; H^{\infty}\left(\mathbb{R}_{t} \times \mathbb{R}_{x^{\prime}}^{n-1}\right)\right)$. This means that $\left(D_{t}^{2}-L\right) \mathcal{R}_{\infty}^{j} f \in C^{\infty}(J \times$ $\left.\mathbb{R}_{t} \times \mathbb{R}_{x^{\prime}}^{n-1}\right)$ ) since the coefficient $a_{n n}$ of $D_{x_{n}}^{2}$ is non-degenerate. Furthermore, $\left.\mathcal{R}_{\infty}^{j} f\right|_{x_{n}=0}$ is equal to $f \bmod C^{\infty}$ if $\mathrm{WF}[f]$ satisfies the condition stated in Theorem 6.2.

By Theorem 6.2 we obtain the following corollary.

Corollary 6.3. Let $f$ in (1.1) be non-glancing and be $C^{\infty}$ smooth if $t<t_{0}$. Then, there exists an outgoing solution $u(t, x)$ (unique $\bmod C^{\infty}$ ) of the equation (1.1) considered in $-\infty<t<t_{0}+\delta$ (and $\left.0<x_{n}<\infty\right)$ for some constant $\delta>0$, and $\operatorname{sing} \operatorname{supp}[u]$ (in $\left.-\infty<t<t_{0}+\delta\right)$ is contained in $\left\{(t, x) \mid t_{0} \leq t<t_{0}+\delta, 0 \leq x_{n} \leq \tilde{\delta}\left(t-t_{0}\right)\right\}$ for a constant $\tilde{\delta}(>0)$. Furthermore, information of $\overline{\mathrm{WF}}[u]$ is obtained by $\mathcal{R}_{\infty}^{\bar{j}} f(j=1, \ldots, d)$. 
Theorem 6.2 implies that for $j=1, \ldots, k, \operatorname{sing} \operatorname{supp} \mathcal{R}_{N}^{j} f$ in $0 \leq x_{n} \leq r(r>0)$ is contained in $\left\{\left(t, x^{\prime}, x_{n}\right) \mid t \geq t_{0}-2^{-1}\left(\partial_{\xi_{n}} \lambda_{j}\left(\tilde{x}^{\prime}, 0, \tilde{\xi}^{\prime} / \tilde{\sigma}, z_{+}^{j}\right)\right)^{-1} x_{n}\right\}$ if sing supp $[f]$ is in $t_{0} \leq t<+\infty$ and $r(>0)$ is small enough. Therefore, in $-\infty<t \leq t_{0}+\delta$, we have $\operatorname{sing} \operatorname{supp} \mathcal{R}_{N}^{j} f \subset\left\{\left(t, x^{\prime}, x_{n}\right) \mid 0 \leq x_{n} \leq-2 \partial_{\xi_{n}} \lambda_{j} \delta\right\} \subset\left\{\left(t, x^{\prime}, x_{n}\right) \mid 0 \leq x_{n} \leq r-\varepsilon\right\}$ for an $\varepsilon>0$ if $\delta$ is small enough, and consequently we can extend $\mathcal{R}_{N}^{j} f C^{\infty}$-smoothly for $x_{n} \geq r-\varepsilon$. Thus we can see that Corollary 6.3 follows from Theorem 6.2.

Proof of Theorem 6.2. The term of the order $\sigma^{-m}$ in $\mathcal{R}_{N}^{j} f$ is of the form

$$
\begin{aligned}
& \sum_{l=1}^{d} \operatorname{Op}\left[\int_{c_{+}} e^{i \sigma x_{n}\left(z+\psi^{j l}\right)} g^{l}\left(I-L_{0}(z)\right)^{-1} h_{m}^{j} \sigma^{-m} \bar{d} z\right] f \\
& \quad=\iint e^{i\left\{t \sigma+x^{\prime} \xi^{\prime}+\sigma x_{n}\left(z_{+}^{j}+\varphi^{j}\right)\right\}} h_{m}^{j}\left(x, \xi^{\prime} / \sigma\right) \sigma^{-m} \chi\left(x, \sigma, \xi^{\prime} / \sigma\right) \hat{f}\left(\sigma, \xi^{\prime}\right) \bar{d} \sigma \bar{d} \xi^{\prime},
\end{aligned}
$$

where $z_{+}^{j}=z_{+}^{j}\left(x, \xi^{\prime} / \sigma\right)$ and $\varphi^{j}=\varphi^{j}\left(x, \xi^{\prime} / \sigma\right)$. Let $\left(t, x^{\prime}, \tilde{\xi}^{\prime} / \tilde{\sigma}, x_{n}\right)$ be in $\mathbb{R} \times U^{\prime} \times J=\mathbb{R} \times U$ (where $U^{\prime}$ and $J$ are the neighborhood and the interval stated in Theorems 4.2 and 5.1), and set

$$
\phi\left(t, x^{\prime}, \tilde{\sigma}, \tilde{\xi}^{\prime}, x_{n}\right)=t \tilde{\sigma}+x^{\prime} \tilde{\xi}^{\prime}+\tilde{\sigma} x_{n} \psi^{j}\left(x^{\prime}, \tilde{\xi}^{\prime} / \tilde{\sigma}, x_{n}\right),
$$

where $\psi^{j}\left(x^{\prime}, \eta^{\prime}, x_{n}\right)=z_{+}^{j}\left(x, \eta^{\prime}\right)+\varphi^{j}\left(x, \eta^{\prime}\right)\left(x=\left(x^{\prime}, x_{n}\right), \eta^{\prime}=\tilde{\xi}^{\prime} / \tilde{\sigma}\right)$. Then we have

$$
\left(\partial_{\tilde{\sigma}} \phi, \partial_{\tilde{\xi}} \phi\right)=\left(t+x_{n} \psi^{j}-x_{n} \partial_{\eta^{\prime}} \psi^{j} \cdot\left(\tilde{\xi}^{\prime} / \tilde{\sigma}\right), x^{\prime}+x_{n} \partial_{\eta^{\prime}} \psi^{j}\right) .
$$

Put $\tilde{t}=\partial_{\tilde{\sigma}} \phi$ and $\tilde{x}^{\prime}=\partial_{\tilde{\xi}^{\prime}} \phi$. Then, if $J$ is small enough, the mapping: $\left(t, x^{\prime}\right) \mapsto\left(\tilde{t}, \tilde{x}^{\prime}\right)$ is invertible. Furthermore $\phi\left(t, x^{\prime}, \sigma, \xi^{\prime}, x_{n}\right)$ satisfies (6.1) for $X=\left(t, x^{\prime}\right)$ and $\Xi=(\sigma, \xi)$.

Since the wave front set of the integral (6.4) (when $x_{n} \in J$ ) has no point if $\left(x^{\prime}, \xi^{\prime} / \sigma\right) \notin$ $U^{\prime}$, we have only to examine the case that $\left(x, \xi^{\prime} / \sigma\right) \in U$. Noting this and setting $X=\left(t, x^{\prime}\right), \tilde{X}=\left(\tilde{t}, \tilde{x^{\prime}}\right)\left(=\left(\partial_{\tilde{\sigma}} \phi, \partial_{\tilde{\xi}^{\prime}} \phi\right)\right), \Xi=\left(\sigma, \xi^{\prime}\right)\left(=\left(\partial_{t} \phi, \partial_{x^{\prime}} \phi\right)\right)$ and $\tilde{\Xi}=\left(\tilde{\sigma}, \tilde{\xi}^{\prime}\right)$, we apply Proposition 6.1 to the integral (6.4). We see that

$$
\begin{aligned}
& t=\tilde{t}-x_{n}\left(\psi^{j}-\partial_{\eta^{\prime}} \psi^{j} \cdot\left(\tilde{\xi}^{\prime} / \tilde{\sigma}\right)\right), \quad \sigma=\tilde{\sigma}, \\
& x^{\prime}=\tilde{x}^{\prime}-x_{n} \partial_{\eta^{\prime}} \psi^{j}, \quad \xi^{\prime}=\tilde{\xi}^{\prime}+\tilde{\sigma} x_{n} \partial_{x^{\prime}} \psi^{j} .
\end{aligned}
$$

Therefore, since $\psi^{j}=z_{+}^{j}+\varphi^{j}$ and $\left.\varphi^{j}\right|_{x_{n}=0}=0$, we have

$$
\begin{aligned}
& \left(t, x^{\prime}, \sigma, \xi^{\prime}\right) \\
& =\left(\tilde{t}, \tilde{x}^{\prime}, \tilde{\sigma}, \tilde{\xi}^{\prime}\right)-x_{n}\left(z_{+}^{j}-\partial_{\eta^{\prime}} z_{+}^{j} \cdot\left(\tilde{\xi}^{\prime} / \tilde{\sigma}\right), \partial_{\eta^{\prime}} z_{+}^{j}, 0,-\tilde{\sigma} \partial_{x^{\prime}} z_{+}^{j}\right)+\left(O\left(x_{n}^{2}\right), O\left(x_{n}^{2}\right), 0, \tilde{\sigma} O\left(x_{n}^{2}\right)\right) .
\end{aligned}
$$

Differentiating the equation $\lambda_{j}\left(x, \eta^{\prime}, z_{+}^{j}\left(x, \eta^{\prime}\right)\right)=1$ in $x^{\prime}$ and $\eta^{\prime}$, we have $\partial_{x^{\prime}} \lambda_{j}+$ $\partial_{\xi_{n}} \lambda_{j} \partial_{x^{\prime}} z_{+}^{j}=0$ and $\partial_{\eta^{\prime}} \lambda_{j}+\partial_{\xi_{n}} \lambda_{j} \partial_{\eta^{\prime}} z_{+}^{j}=0$. Since $\lambda_{j}(x, \xi)$ is homogeneous of order 2 in $\xi$, it follows (from the Euler equality) that $\partial_{\eta^{\prime}} \lambda_{j} \cdot \eta^{\prime}+\left(\partial_{\xi_{n}} \lambda_{j}\right) z_{+}^{j}=2$. Therefore we obtain $-\left(\partial_{\xi_{n}} \lambda_{j}\right) \partial_{\eta^{\prime}} z_{+}^{j} \cdot \eta^{\prime}+\left(\partial_{\xi_{n}} \lambda_{j}\right) z_{+}^{j}=2$, which yields that $z_{+}^{j}-\partial_{\eta^{\prime}} z_{+}^{j} \cdot\left(\tilde{\xi}^{\prime} / \tilde{\sigma}\right)=2\left(\partial_{\xi_{n}} \lambda_{j}\right)^{-1}$. Therefore, noting that $\partial_{\eta^{\prime}} z_{+}^{j}=-\left(\partial_{\xi_{n}} \lambda_{j}\right)^{-1} \partial_{\eta^{\prime}} \lambda_{j}$ and $\partial_{x^{\prime}} z_{+}^{j}=-\left(\partial_{\xi_{n}} \lambda_{j}\right)^{-1} \partial_{x^{\prime}} \lambda_{j}$, we get 


$$
\begin{aligned}
& \left(t, x^{\prime}, \sigma, \xi^{\prime}\right) \\
& \quad=\left(\tilde{t}, \tilde{x}^{\prime}, \tilde{\sigma}, \tilde{\xi}^{\prime}\right)-x_{n}\left(\partial_{\xi_{n}} \lambda^{j}\right)^{-1}\left(2,-\partial_{\eta^{\prime}} \lambda^{j}, 0, \tilde{\sigma} \partial_{x^{\prime}} \lambda^{j}\right)+\left(O\left(x_{n}^{2}\right), O\left(x_{n}^{2}\right), 0, \tilde{\sigma} O\left(x_{n}^{2}\right)\right) .
\end{aligned}
$$

Hence we obtain (i) of Theorem 6.2.

(ii) of Theorem 6.2 follows from Theorem 4.2. Thus Theorem 6.2 is proved.

\section{References}

[1] K. O. Friedrichs, Symmetric hyperbolic system of linear differential equations, Comm. Pure Appl. Math., 7 (1954), 345-392.

[2] M. Kawashita, H. Soga and J. Ralston, Complex analysis of elastic symbols and construction of plane wave solutions in the half-space, J. Math. Soc. Japan, 55 (2003), 395-404.

[ 3 ] H. Kumano-go, Pseudo-differential operators, MIT Press, Cambridge, 1981.

[4] P. Lax, Asymptotic solutions of oscillatory initial value problems, Duke Math. J., 24 (1957), 627-646.

[5] H. Soga, Asymptotic solutions of the elastic wave equation and reflected waves near boundaries, Comm. Math. Phys., 133 (1990), 37-52.

[6] H. Soga, Asymptotic solutions of the elastic wave equation in the case of total reflection, Comm. PDE, 26 (2001), 2249-2266.

[ 7 ] M. Taylor, Pseudodifferential Operators, Princeton Univ. Press, Princeton, 1981.

\section{Hideo SogA}

College of Education

Ibaraki University

Mito

Ibaraki 310-8512, Japan

E-mail: hideo.soga.310@vc.ibaraki.ac.jp 